\title{
Device Efficiency of Organic Light-Emitting Diodes: Progress by Improved Light Outcoupling
}

\author{
Wolfgang Brütting*, Jörg Frischeisen, Tobias D. Schmidt, Bert J. Scholz, and Christian Mayr
}

Institute of Physics, University of Augsburg, 86135 Augsburg, Germany

Received XXXX, revised XXXX, accepted XXXX

Published online XXXX

Key words: organic LEDs, efficiency, optical simulation.

* Corresponding author: e-mail wolfgang.bruetting@physik.uni-augsburg.de, Phone: +49-821-5983403, Fax: +49-821-5983425

Organic light-emitting diodes (OLEDs) are efficient large-area light sources facing their market entry. Still, the development of stable and more efficient blue emitters and the enhancement of light outcoupling remain challenges for further device improvements. Here, we review the working principles of OLEDs and highlight ongoing efforts to improve their efficiency, in particular by coupling out more light.

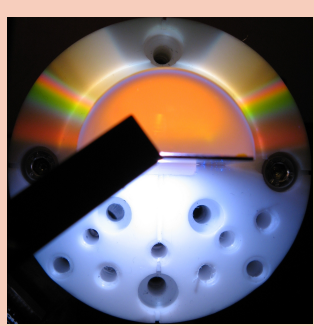

Photograph of a white light emitting OLED showing direct light-emission to the bottom side, as well as light emission from trapped modes to the top side.

Copyright line will be provided by the publisher

1 Introduction The availability of artificial light has been a seminal cultural development of mankind. After open fires for thousands of years, the introduction of electricity together with the invention of the light bulb in the second half of the 19th century has revolutionized our daily life. Having dominated the lighting market for more than 100 years, however, the light bulb faces its decline due to the need for technologies that convert electricity more efficiently into visible light. Besides fluorescent lamps, which are already well established in the market, the availability and progress in white light-emitting diodes, both inorganic and organic, over the last decade has lead to a new lighting technology called solid-state lighting [1]. Its working principle, namely the radiative recombination of injected electron-hole pairs in a solid, a process termed electroluminescence, is fundamentally different from thus far existing techniques and holds the promise for highly efficient, long-lived and environmentally friendly light sources. In contrast to their inorganic counterparts, organic light- emitting diodes (OLEDs) are flat and thin large-area light sources that could rather lead to complementary luminaires than competitors to existing technologies.

Historically speaking, electroluminescence in organic molecular crystals dates back to the early 1960s [2,3], however, the important step towards applicable devices was the demonstration of thin film organic light-emitting diodes by researchers at Eastman Kodak in 1987 [4] using vacuum deposited molecular materials and by a group at Cambridge University in 1990 [5] making use of a solution processed conjugated polymer. Inspired by these publications, intense research and development has lead to the introduction of first commercial products based on OLED displays in the late 1990s. Since 2000, however, the focus in many laboratories shifted towards OLEDs for lighting applications, i.e. to white OLEDs. After steady improvements in efficiency and lifetime over the years, the commercialization of OLEDs for general lighting has just recently started [6]. 


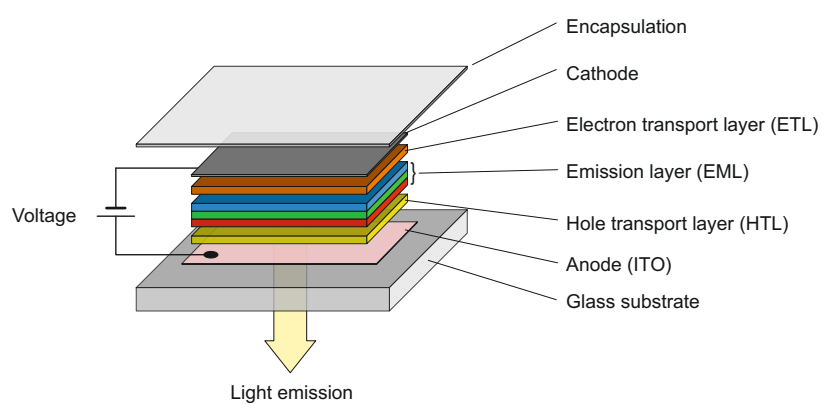

Figure 1 Simplified illustration of a three-layer OLED stack. ETL, EML and HTL denote the electron transport layer, the emission layer(s) and the hole transport layer, respectively. In order to obtain light emission, a positive voltage must be applied to the anode, which often consists of optically transparent indium-tin oxide (ITO).

The scope of this article is to present the working principles of OLEDs and to review ongoing efforts to improve their efficiency, in particular through enhanced light outcoupling. Thereby we will restrict ourselves to devices fabricated by vacuum deposition of low-molecular weight materials (small molecules). Nevertheless, most of the following discussion is equally valid for polymer OLEDs where thin films are prepared by solution processing of large macromolecules. For an overview of various technical aspects of OLED fabrication and the current state of the art of white OLEDs for lighting applications see, e.g., Ref. [7$11]$.

\section{OLED Operation}

2.1 OLED Architecture and Stack Layout In principle, an OLED can consist of only one organic layer sandwiched between two electrodes, i.e. the anode and the cathode [5]. However, a multilayer OLED made of several different functional organic layers is superior in terms of efficiency and lifetime because the properties of the individual layers can be tuned through the proper choice of materials. A typical stack layout of such an OLED is displayed in Fig. 1. The positively biased anode is required in order to inject holes into the hole transport layer (HTL). Similarly, electrons are injected from the cathode into the electron transport layer (ETL). The emission layer (EML) comprises one or more dyes emitting light in different regions of the visible spectrum. High-efficiency OLEDs often contain several additional layers like separate blocking and injection layers.

The most common substrate material is glass, but it is also possible to employ other materials, like polymer or metal foils or semiconductor substrates. One of the two metallic or metallic-conducting electrodes must be semitransparent so that the produced light can leave the device either to the substrate side (bottom emission) or through the upper electrode (top emission). Frequently, the anode of an OLED consists of indium-tin oxide (ITO), which is highly transparent and shows a good electrical conductivity. In general, anode materials need a high work function which also makes metals like gold a suitable choice. For the cathode, low work function metals like calcium or magnesium are often used in combination with highly reflective and less reactive metals like aluminum or silver.

Since many organic materials and low work function metals are not stable under ambient conditions [12], i.e. they react with oxygen and moisture, it is necessary to protect the materials with an encapsulation. The most common method utilizes a cover glass that is glued to the substrate, thus creating a cavity filled with inert gas or a desiccant acting as a getter to absorb small amounts of oxygen and water that penetrate through the encapsulation in the course of time. The active area of the OLED is defined as the overlap of the two electrodes. Hence, OLEDs can in principle have any shape and size and they are therefore suited for a wide range of applications. Laboratory OLEDs have typical dimensions of a few $\mathrm{mm}^{2}$. In industrial prototypes the active area of the OLEDs has already been scaled-up to sizes in the range 0.1-1 square meters. The thickness of the organic layers is typically in the range between 10 and a few hundred nanometers.

2.2 Working Principles of OLEDs The basic processes in an OLED under operation are illustrated in Fig. 2 for a device with three organic layers. An external voltage source of typically a few volts is applied to the device so that the two types of charge carriers are injected from the opposite electrodes, i.e. electrons from the cathode and holes from the anode, and drift towards each other. When the initially free electrons and holes meet, they form strongly bound electron-hole pairs (excitons) in the emission layer, which then may decay radiatively and emit photons. In detail, the whole process can be separated into four fundamental steps as denominated in Fig. 2:

(1)Injection of electrons and holes at the electrodes

(2)Transport of charge carriers through the organic layers

(3)Formation of bound electron-hole pairs (excitons)

(4)Radiative exciton decay and emission of light

One distinct difference between OLEDs based on molecular materials and polymer OLEDs is the extend and location of the recombination zone inside the device. While in the latter case the emission zone is spread over a sizeable fraction of the light-emitting polymer layer (depending of course on the detailed material properties) [17], in small molecule OLEDs the generation of light can be confined by a proper choice of materials with suitable energy levels and layer thicknesses to a relatively narrow zone sandwiched between HTL and ETL. Moreover, if doped hole and electron injection layers are used [18], this region can be placed at almost arbitrary distance away from the electrodes. This is favorable because recombination near the electrodes usually causes quenching and therefore a reduction in efficiency. Further on, as will be discussed later in this article, this additional degree of free- 


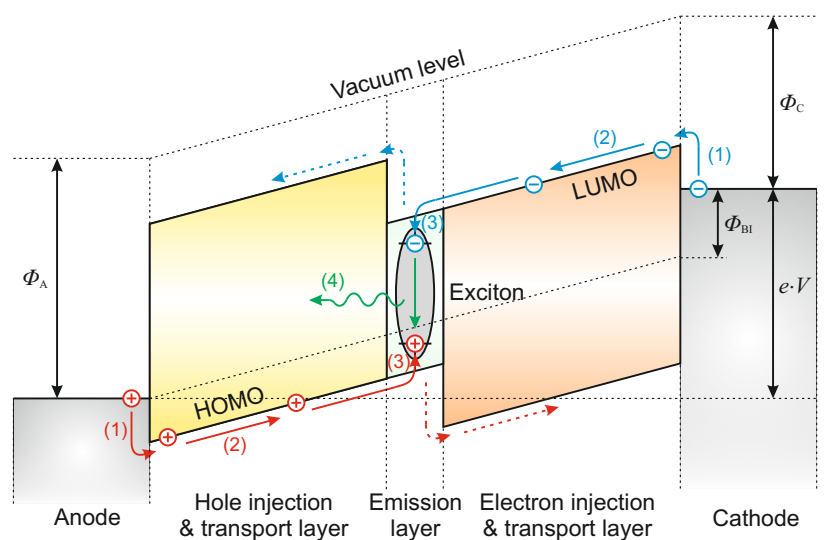

Figure 2 Schematic energy diagram of a three-layer OLED illustrating the basic processes of electroluminescence. Electrons are injected from the cathode into the lowest unoccupied molecular orbital (LUMO) of the ETL, holes from the anode into the highest occupied molecular orbital (HOMO) of the HTL. Since the anode and cathode have different work functions $\Phi_{\mathrm{A}}$ and $\Phi_{\mathrm{C}}$, respectively, a built-in voltage $\Phi_{\mathrm{BI}}$ exists in the device, which has to be overcome by an external voltage $V$ before current can flow through the device. If materials are properly chosen, recombination of electrons and holes will be confined to the EML and the flow of excess carriers not recombining will be minimized. It should be noted that owing to disorder the energy levels of all involved states are not discrete but distributed in energy, thus leading to important consequences for charge carrier transport and recombination. (For further details on this aspect, we refer to the literature, e.g. $[13,14]$.) In addition, the possibility of trap-assisted recombination (e.g. [15]) is not included in this diagram. Furthermore, vacuum level shifts and other effects occurring at interfaces between different materials (see e.g. [16]) are not taken into account.

dom is a handle to improve OLEDs with respect to light outcoupling by making use of optical interference effects.

2.3 OLED Materials The development of OLEDs has been enabled by and will continue to rely on the availability of tailor-made functional organic materials that can be applied to well-controlled thin films. Thereby the requirements to the materials are manifold: starting from processibility and film formation, via electrical transport to optical properties. The key factor is obviously the availability of efficient and stable light emitters in the full visible spectral range. In this respect one has to distinguish between fluorescent and phosphorescent materials. A seminal step was the introduction and further development of emitters based on heavy-metal centred metal-organic complexes, as shown in Fig. 3. In these compounds strong spin-orbit coupling mixes singlet and triplet states much more than in pure hydrocarbons, so that phosphorescence becomes an allowed transition [19]. In the meantime, impressive efficiency data have been published for OLEDs based on these materials $[11,20,21]$, however, the bottleneck is still the

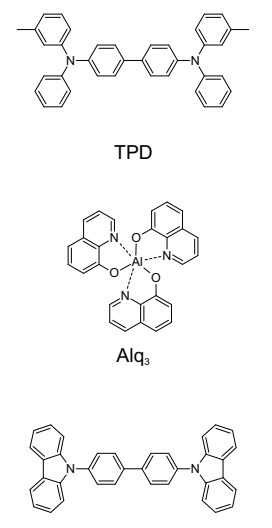

CBP
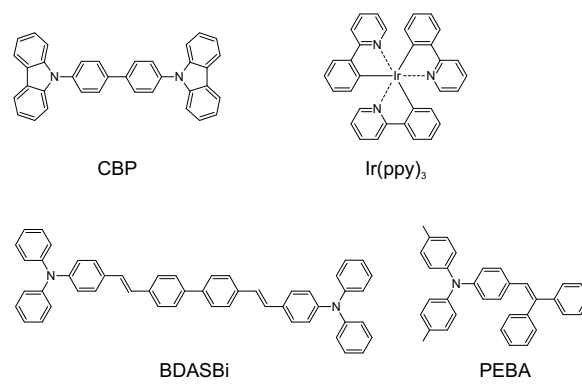

$\operatorname{Ir}(\text { ppy })_{3}$

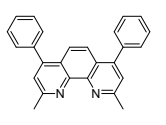

$\mathrm{BCP}$
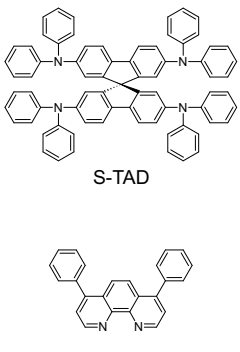

BPhen

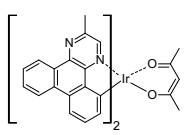

$\operatorname{Ir}(\mathrm{MDQ})_{2}(\mathrm{acac})$

Figure 3 Selection of organic materials used for OLEDs that are discussed in this article. TPD, $\alpha$-NPD and S-TAD are hole transport materials. $\mathrm{Alq}_{3}, \mathrm{BCP}$ and BPhen are electron transport materials, with $\mathrm{Alq}_{3}$ often being used as green fluorescent emitter material, too. CBP serves as matrix material for the green phosphorescent emitter $\operatorname{Ir}(\mathrm{ppy})_{3}$ or the two blue fluorescent emitters BDASBi and PEBA. The red phosphorescent emitter $\operatorname{Ir}(\mathrm{MDQ})_{2}$ (acac) is doped into an $\alpha$-NPD host layer. PEDOT:PSS is a conducting polymer that can be used as hole injection layer, because it effectively increases the work function of ITO and serves as a planarization layer.

limited availability and stability of deep-blue phosphorescent emitters.

A selection of common organic materials that were used for fabricating OLEDs in our group are displayed in Fig. 3. Fig. 4 shows electroluminescence characteristics of two prototypical OLEDs, one with the fluorescent emitter $\mathrm{Alq}_{3}$ and the other one with the phosphorescent material $\operatorname{Ir}(\mathrm{ppy})_{3}$. Both will serve as reference devices throughout this article.

2.4 White OLEDs One of the peculiarities of organic emitters is their intrinsically broad luminescence spectrum. By the combination of several organic emitters with different emission colour it is possible to generate light spreading over the whole visible spectral range, thus having an excellent colour rendering index (CRI), i.e. the ability to reproduce the colour of illuminated objects. There are several methods in order to create such white OLEDs. The first report dates back to 1994, when a Japanese group combined red, green and blue (RGB) laser dyes in a common matrix and achieved light emission over a broad spectral range [22]. Despite its simplicity in preparation, achieving white light emission with good and stable colour quality in this way is not that straightforward, because charge recom- 

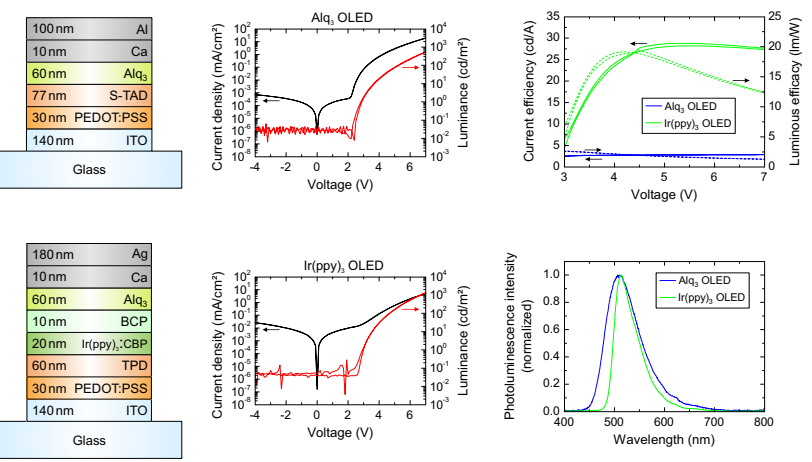

Figure 4 Layer structures of two prototypical OLEDs with $\mathrm{Alq}_{3}$ as fluorescent and $\operatorname{Ir}(\mathrm{ppy})_{3}$ as phosphorescent green emitters, respectively. Also shown are their current-voltage-luminance characteristics as well as their electroluminescence emission spectra and their efficiency.

bination and energy transfer processes between the different dyes need to be well controlled. Thus it is nowadays more common to employ distinct emission layers for RGB that can either be directly stacked on top of each other in one OLED (see Fig. 1) or in three separated OLEDs - one for each colour - that are in turn vertically stacked by optically transparent electrical interconnecting units. In addition, white light from OLEDs may be produced similar to inorganic LEDs, i.e. by combining a blue OLED with either red and green or yellow colour conversion layers [23]. Depending on the method and the choice of materials it is thus possible to cover a large variety of colours and colour temperatures within the CIE 1931 colour space (Commission internationale de l'éclairage). Further details about different concepts of white OLEDs and the current state of the art can be found in Refs. [8-11].

\section{Electroluminescence Quantum Efficiency}

3.1 Factors Determining the EQE Apart from its spectral characteristics, the most important parameter characterizing an OLED is the external electroluminescence quantum efficiency (EQE) that describes the ratio between the number of emitted photons and injected charge carriers [24]:

$$
\eta_{\mathrm{EQE}}=\gamma \cdot \eta_{\mathrm{S} / \mathrm{T}} \cdot q_{\mathrm{eff}} \cdot \eta_{\mathrm{out}} \cdot
$$

Therein $\gamma$ is the charge carrier balance factor, describing whether or not equal amounts of electrons and holes are injected and what fraction of them recombines to form an exciton. The second factor $\eta_{\mathrm{S} / \mathrm{T}}$ gives the fraction of excitons that is allowed to decay radiatively by spin statistics. The third factor $q_{\mathrm{eff}}$ indicates how many of the spin-allowed excitons actually do decay by emitting a photon (instead of dissipating the excitation energy nonradiatively to their environment). Finally, the last factor $\eta_{\text {out }}$ determines which fraction of the generated photons are in the end able to leave the device to the outside world. Hence the external quantum efficiency can be split into an internal quantum efficiency $\eta_{\mathrm{IQE}}=\gamma \cdot \eta_{\mathrm{S} / \mathrm{T}} \cdot q_{\mathrm{eff}}$ times the outcoupling factor $\eta_{\text {out }}$.

In detail (see Fig. 2), the charge carrier balance $\gamma$ depends on the numbers of electrons and holes that are injected and the fraction of them, which is consumed by recombination:

$$
\gamma=\frac{j_{\mathrm{R}}}{j_{\mathrm{tot}}}
$$

with $j_{\text {tot }}=j_{\mathrm{h}}+j_{\mathrm{e}}^{\prime}=j_{\mathrm{e}}+j_{\mathrm{h}}^{\prime}$ being the total current density and $j_{\mathrm{R}}=j_{\mathrm{h}}-j_{\mathrm{h}}^{\prime}=j_{\mathrm{e}}-j_{\mathrm{e}}^{\prime}$ the recombination current density. Therein $j_{\mathrm{h}, \mathrm{e}}$ are the injected hole and electron currents, respectively, and the primed quantities are the corresponding fractions of carriers leaving the device at the opposite electrode without recombination. Under ideal conditions the latter will be zero and consequently $\gamma=1$. However, if there is an imbalance of the numbers of injected electrons and holes or if recombination is not complete then there is excess of charge carriers that does not contribute to the production of light, and the efficiency of the OLED is reduced. In small molecule multilayer OLEDs the charge carrier balance can be brought close to unity by using doped transport layers and additional selective carrier and exciton blocking layers $[18,25]$.

The so-called singlet/triplet factor $\eta_{\mathrm{S} / \mathrm{T}}$ describes the probability for the formation of an exciton that is allowed to decay radiatively according to the spin selection rules. Under electrical operation, singlet and triplet excitons are formed by recombination of electrons and holes, each of them carrying spin $\frac{1}{2}$. The probability of forming a triplet exciton with total spin $S=1$ is statistically three times higher than the probability of forming a singlet exciton with $S=0$, because the spin orientations of the injected electrons and holes are random and every triplet state is threefold degenerate. Therefore, $\eta_{\mathrm{S} / \mathrm{T}}$ is believed to have a value of $25 \%$ for fluorescent emitters which only make use of singlet excitons, while it can be $100 \%$ for phosphorescent emitters, where both singlets and triplets contribute to light emission [26,27].

We note that even for singlet emitters $\eta_{\mathrm{S} / \mathrm{T}}$ might be enhanced beyond $25 \%$ by triplet-triplet annihilation (TTA) [28-31] as well as thermally activated triplet-to-singlet up-conversion $[32,33]$ in certain material systems. In the context of conjugated polymer systems, there has been a debate whether $\eta_{\mathrm{S} / \mathrm{T}}$ might intrinsically be larger than $25 \%$ (even in the absence of TTA) [34-36], however, recent results indicate that this is probably not the case [37]. As will be discussed below, an unambiguous assignment requires a careful efficiency analysis including effects of non-isotropic orientation of the light-emitting chromophores. All in all, a significant efficiency enhancement can be obtained by using phosphorescent materials, hence $\eta_{\mathrm{S} / \mathrm{T}}$ can be raised to $1[19,38]$.

The effective radiative quantum efficiency $q_{\text {eff }}$ is derived from the intrinsic radiative quantum efficiency $q$ of the emitter material, which is obtained if the emitting species is surrounded by an unbounded medium in the 
limit of very low excitation densities. Usually the photoluminescence quantum efficiency measured by using a thin film of the emitter embedded in the same matrix as in the OLED comes close to this value. Per definition $q$ is given as

$$
q=\frac{\Gamma_{\mathrm{r}}}{\Gamma_{\mathrm{r}}+\Gamma_{\mathrm{nr}}},
$$

where $\Gamma_{\mathrm{r}}$ is the radiative decay rate of the excited state and $\Gamma_{\mathrm{nr}}$ is the sum of all competing non-radiative decay rates. It is apparent that every non-radiative contribution to the exciton decay reduces the radiative quantum efficiency. In addition, in an OLED environment the presence of stratified media with different refractive indices as well as the vicinity to metallic electrodes lead to a modification of the radiative decay rate of an exciton via the Purcell effect: $\Gamma_{\mathrm{r}} \rightarrow \Gamma_{\mathrm{r}}^{*}=F \cdot \Gamma_{\mathrm{r}}$ (with $F$ being the Purcell factor) [3941]. By contrast, the non-radiative decay rates, e.g. the dissipation of excitation energy into heat, are not affected by the cavity environment [42]. Thus, the effective radiative quantum efficiency $q_{\text {eff }}$ can be defined as

$$
q_{\mathrm{eff}}=\frac{\Gamma_{\mathrm{r}}^{*}}{\Gamma_{\mathrm{r}}^{*}+\Gamma_{\mathrm{nr}}}=\frac{F \cdot \Gamma_{\mathrm{r}}}{F \cdot \Gamma_{\mathrm{r}}+\Gamma_{\mathrm{nr}}} .
$$

Depending on the details of the layer stack, the cavity effect can either increase or reduce $q_{\mathrm{eff}}$ with respect to the intrinsic value $q$. Consequently, the optimization of the OLED cavity is very important not only in terms of the light outcoupling efficiency, as will be discussed below, but also to enhance the radiative decay processes in cases where the emitter does not a priori have $q=1$. And even if $q$ is close to 1 , one has to be aware that in an operating device the radiative quantum efficiency can be significantly reduced by bimolecular quenching of excitons or interactions with charge carriers at high current densities [43]. This is especially relevant for long-lived triplet excitons (triplet-triplet quenching, triplet-polaron quenching) as will be shown later on.

With the above said, the internal quantum efficiency of OLEDs can be brought up towards the theoretical limit of $100 \%$, if charge carrier injection and recombination are well balanced, if phosphorescent emitters are used and if non-radiative exciton quenching processes are suppressed [25]. Nevertheless, only a fraction of the light will in the end be able to leave the device to the outside world. The reason is that light is generated in a region of the OLED stack with higher refractive index than the glass substrate and, obviously, ambient air. In a simple model based on ray optics the light outcoupling efficiency is given by [44]:

$$
\eta_{\mathrm{out}}=\frac{1}{2 n^{2}}
$$

where $n$ denotes the (average) refractive index of the organic layer stack. With typical values of $n=1.6-1.8$ one immediately finds that only between 15 and $20 \%$ of the optical power is actually extracted from an OLED. However,

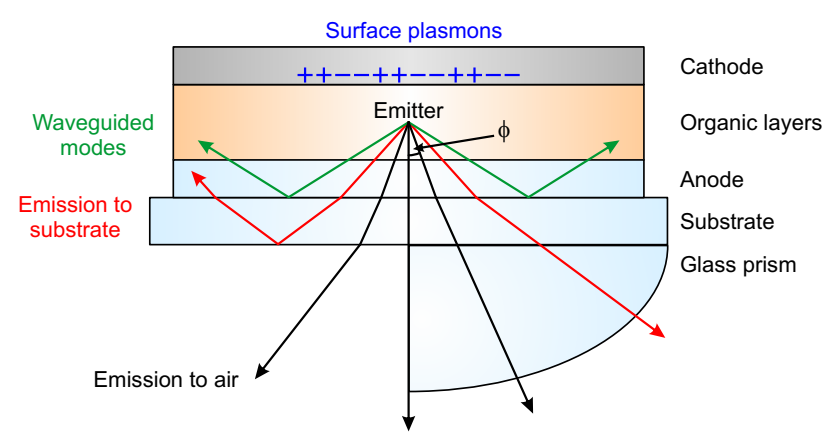

Figure 5 Schematic illustration of an OLED showing different optical loss channels. Without outcoupling enhancement, only a small fraction of light is directly emitted to air as shown in the left part of the figure. By applying a macro-extractor, e.g. an indexmatched glass hemisphere, also the substrate emission can be extracted (cf. the right half of the figure).

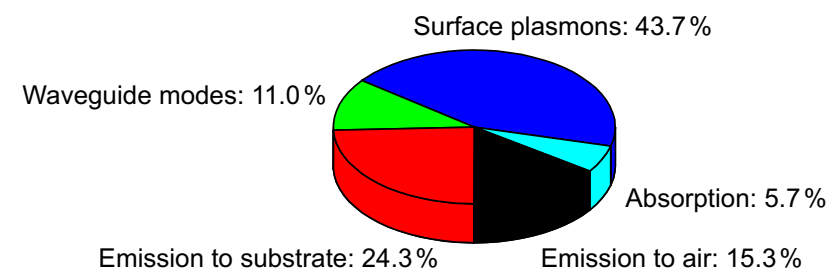

Figure 6 Amount of power coupled to different optical channels in the prototypical Alq $\mathrm{ALED}_{3}$ from Fig. 4. The numbers are obtained by integrating each region in the power dissipation spectrum shown in Fig. 8(a). In this diagram, a radiative quantum efficiency of $q=1$ is assumed. The influence of $q$-values below unity are discussed in the text.

this should only be taken as a rough estimation; a more sophisticated analysis has to take the coupling of the excited molecules to the modes of the OLED cavity into account, as will be discussed in detail in the next section.

\section{Fundamentals of Light Outcoupling in OLEDs}

4.1 Optical Loss Channels As already mentioned above, a ray optical model can only give a rough estimate of the light extraction efficiency $\eta_{\text {out }}$ of an OLED. For a more accurate treatment wave optical methods are required, which will be addressed in this section. An excited molecule can couple to different optical modes in such a thin film structure (see Fig. 5). Viewed from the emitter position the light escape cone has an opening angle of some $30^{\circ}$ with respect to the surface normal and the energy contained in it typically amounts to less than $20 \%$ of the total energy. This is followed by the contribution of substrate modes, where total internal reflection at the glass-air interface is the limiting process. This contribution is comparable in energy also at around 20\%. For higher emission angles the light can not even reach the glass substrate, but is wave-guided in the organic layers (including the transparent indium-tin oxide electrode) and 
in the end lost by residual absorption or edge emission. Finally, the emitter can couple to the evanescent field of surface plasmon polaritons (SPP) travelling at the interface between the metal electrode and the organic layers. Quantitative calculations, treating the emitting molecules as classical electrical dipoles (for details see below), reveal that in planar OLED stacks typically around $50 \%$ of the light is trapped in waveguided and plasmon modes $[40,45,46]$. As an example, Fig. 6 shows the contribution of different optical channels for the prototypical $\mathrm{Alq}_{3}$ OLED stack discussed before in Fig. 4. Note that this chart is obtained with a radiative quantum efficiency $q=1$. It thus describes the maximum light outcoupling of the stack under ideal conditions. The given numbers are valid regardless if the emitter is fluorescent (e.g. $\mathrm{Alq}_{3}$ ) or phosphorescent (e.g. $\left.\operatorname{Ir}(\mathrm{ppy})_{3}\right)$, as long as the layer stack has comparable thicknesses and refractive indices and the emission spectra are not too different. Given the low number of directly emitted light, it is therefore not surprising that developing new concepts for improving light extraction efficiency has been a major issue over recent years (for an overview see, e.g. Ref. [47]). Some of these approaches (including our own results) will be presented in section 5 .

4.2 Optical Modeling of OLEDs Fluorescence and phosphorescence of excited molecules are not intrinsic properties of a material, but are modified by the optical environment of the emitting species. Originally discovered for magnetic resonance, this so-called Purcell effect [48] has been found to be relevant also in experiments with fluorescent molecules near interfaces $[49,50]$. After its experimental demonstration Chance, Prock and Silbey (CPS) developed a theoretical framework by applying the classical theory of an oscillating electrical dipole near a dielectric interface to the problem of molecules fluorescing near a surface [51,52]. Thereby they made use of the fact that the probability for the emission of a photon by an excited molecule via a dipole transition is equivalent to the power radiated by a classical dipole antenna. The formalism has later on been extended to structures where the emitter is embedded in a layer stack with multiple interfaces [53] and to microcavity structures $[54,55]$.

Here we follow the approach by Wasey and Barnes [56], where the problem of an incoherent ensemble of dipole emitters being embedded in a planar thin-film structure is solved by a plane wave expansion of the electric field with appropriate consideration of the electromagnetic boundary conditions (see Fig. 7). Formally the whole OLED stack is split into an upper and a lower half with respect to the emitter position and the propagation of radiation in both directions is calculated by taking into account the Fresnel reflection and transmission coefficients at the involved interfaces. Key parameters entering into the model are the position $(z)$ of the emitter with respect to interfaces (in particular the highly reflective cathode in bottom emitting structures), the emitter orientation $(\Theta)$, the thicknesses $(d)$ and optical constants (refractive index $n$,

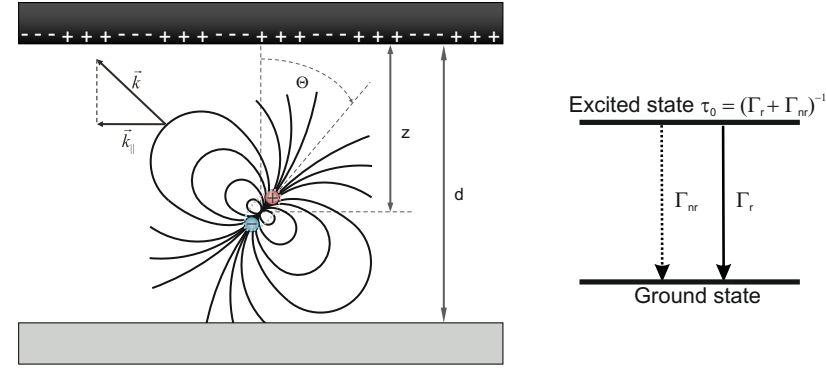

Figure 7 Schematic illustration of an oscillating electrical dipole embedded in a dielectric layer, which is sandwiched between two interfaces. The upper material is assumed to be a metal, so that surface plasmon polaritons are supported at this interface. The other parameters are the layer thickness $d$, the distance $z$ of the dipole to the metal, its relative orientation $\Theta$ to the surface normal, the wave vector $\mathbf{k}$ and its in-plane component $\mathbf{k}_{\|}$. The quantum mechanical analogue is a two-level system consisting of the energy levels of the excited state of the molecule and the ground state with radiative and non-radiative decay rates between them, leading to a finite lifetime of the excited state. Note that if the dipole is embedded in a cavity, the radiative rate will be modified to become $\Gamma_{\mathrm{r}}^{*}=F \cdot \Gamma_{\mathrm{r}}$ (with $F$ being the Purcell factor, see text) and the excited state lifetime accordingly $\tau=\left(\Gamma_{\mathrm{r}}^{*}+\Gamma_{\mathrm{nr}}\right)^{-1}$.

extinction coefficient $\kappa$ ) of all involved layers and the intrinsic radiative quantum efficiency $(q)$ of the emitter (often embedded in a matrix material). The presence of the OLED cavity now has a twofold effect on the properties of light emission with respect to the case when the emitter resides in an infinite medium with the same index of refraction: It will modify the radiative decay rate of the emitter (thus leading to an effective radiative quantum efficiency $q_{\text {eff }}$ in Eq. 1) and it will redistribute the optical power between different channels (thus determining the light outcoupling factor $\eta_{\text {out }}$ ).

According to Ref. [56] the relative decay rate $\left(\Gamma / \Gamma_{0}\right)$ of an ensemble of isotropically oriented and mutually incoherent dipoles emitting at a single wavelength $\lambda$ takes the following form:

$$
\tilde{P}=\frac{\Gamma}{\Gamma_{0}}=(1-q)+q \cdot \int_{0}^{\infty} P\left(k_{\|}, \lambda, z\right) d k_{\|} .
$$

Therein $\Gamma=\Gamma_{\mathrm{r}}^{*}+\Gamma_{\mathrm{nr}}$ is the decay rate of the emitter modified by the presence of the cavity, $\Gamma_{0}=\Gamma_{\mathrm{r}}+\Gamma_{\mathrm{nr}}$ is the original decay rate without a cavity, $q$ is the intrinsic radiative quantum efficiency (again for the emitter in a homogeneous unbounded medium) and $P$ is the layer specific power dissipation function depending on the in-plane wave vector $k_{\|}$, the wavelength $\lambda$ and the emitter position within the layer stack denoted by $z$. For the sake of simplicity we will always use a discrete emitter position, i.e. an infinitely sharp recombination zone inside the OLED. This assumption is not so far from reality for small molecule OLEDs, where the exciton formation zone is confined by using a 
(a)
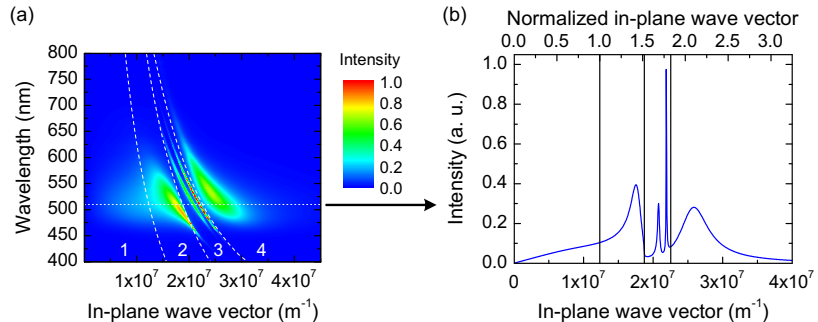

Figure 8 (a) Simulation of the total dissipated optical power for the $\mathrm{Alq}_{3}$ OLED shown in Fig. 4. The emitter position is assumed at the interface between $\mathrm{Alq}_{3}$ and S-TAD. The result is shown for an isotropic dipole orientation. Red and yellow areas indicate a high amount of dissipated power. The dashed white lines divide the graph into four regions: (1) emission into air, (2) emission into substrate, (3) waveguide modes and (4) coupling to surface plasmons. (b) Cross section of (a) at a wavelength of $510 \mathrm{~nm}$, i.e. at the emission maximum. The upper axis shows the normalized in-plane wave vector with respect to the vacuum wave vector at a wavelength of $510 \mathrm{~nm}$. (See also Ref. [40]).

thin dye-doped emission layer surrounded by selective carrier blocking layers. It is, however, quite straightforward to extend the formalism to an extended emission zone by introducing some distribution function in $z$ and averaging over all emitter positions (see e.g. [17,57]). The same holds for the emitter orientation. The calculation is actually performed for the three fundamentally different dipole orientations:

$-P_{\perp, \mathrm{TM}}$ : dipoles oriented perpendicular to the substrate plane (vertical), which emit p-polarized (transverse magnetic (TM)) light

$-P_{\|, \mathrm{TM}}$ : dipoles oriented parallel to the substrate plane (horizontal), which emit p-polarized (transverse magnetic (TM)) light

$-P_{\|, \mathrm{TE}}$ : dipoles oriented parallel to the substrate plane (horizontal), which emit s-polarized (transverse electric (TE)) light

For symmetry reasons, the contributions of the two horizontal dipole components are equal, so that in the case of a random distribution of dipole orientations $P$ is given as $P_{\text {(iso) }}=\frac{1}{3} P_{\perp}+\frac{2}{3} P_{\|}$. If not otherwise stated, we will in the following assume isotropic emitter orientation. The effect of non-isotropic orientation on the light outcoupling efficiency will be discussed separately at the end of section 5 .

Fig. 8 shows the power dissipation spectrum corresponding to the prototypical OLED stack shown in Fig. 4, where the emission is assumed to take place in $\mathrm{Alq}_{3}$ directly at the interface to the HTL. Furthermore we have used a hypothetical radiative quantum efficiency $q=1$ and weighted the simulation results for different wavelengths between 400 and $800 \mathrm{~nm}$ with the normalized photoluminescence spectrum $S(\lambda)$ of the emitter. Also shown in the figure is a cross section at a wavelength of $510 \mathrm{~nm}$, where the different optical loss channels can be clearly identified.
According to Eq. 6 this power dissipation spectrum can now be used to calculate the total relative decay rate by integrating over all relevant wavelengths (between $\lambda_{1}=$ $400 \mathrm{~nm}$ and $\lambda_{2}=800 \mathrm{~nm}$ ) and in-plane wave vectors:

$$
\begin{aligned}
\tilde{P}_{\text {tot }} & =(1-q)+q \cdot \int_{\lambda_{1}}^{\lambda_{2}} S(\lambda) \int_{0}^{\infty} P\left(k_{\|}, \lambda, z\right) d k_{\|} d \lambda \\
& \equiv(1-q)+q \cdot F .
\end{aligned}
$$

The latter equivalence follows from the definition of the decay rates in free space $\Gamma_{0}=\Gamma_{\mathrm{r}}+\Gamma_{\mathrm{nr}}$ and inside the cavity $\Gamma=\Gamma_{\mathrm{r}}^{*}+\Gamma_{\mathrm{nr}}=F \cdot \Gamma_{\mathrm{r}}+\Gamma_{\mathrm{nr}}$. Thus the integral of the spectrally weighted power dissipation directly yields the Purcell factor $F$. With that the effective radiative quantum efficiency of the emitter in the OLED cavity is given by:

$$
\frac{q_{\mathrm{eff}}}{q}=\frac{F \cdot\left(\Gamma_{\mathrm{r}}+\Gamma_{\mathrm{nr}}\right)}{F \cdot \Gamma_{\mathrm{r}}+\Gamma_{\mathrm{nr}}}=F \cdot \frac{\Gamma_{0}}{\Gamma}=\frac{F}{(1-q)+q \cdot F} .
$$

Of course, this concept is equally valid for non-isotropic emitter orientation, if the calculation of the Purcell factor is performed separately for each of the three orthogonal orientations ( $x, y$ being parallel to the substrate plane, and $z$ being perpendicular to it) and by taking deviations from randomness into account via an orientation anisotropy factor $\theta$ so that $F$ in the previous expression is replaced by $F(\theta)$ (for details see Ref. [58]):

$$
F(\theta) \equiv \frac{1-\theta}{2} \cdot\left(F_{x}+F_{y}\right)+\theta \cdot F_{z} .
$$

Thereby $\theta$ denotes the fraction of vertical dipoles: $\theta=1 / 3$ for isotropic orientation, $\theta=0$ for completely horizontal ( $x$ - $y$-plane) orientation and $\theta=1$ for fully vertical $(z-$ direction) emitter orientation, respectively.

We note that in this terminology the coupling of the excited molecules to modes of the cavity that do a priori not radiate into the far-field, such as waveguide modes or surface plasmons, are radiative processes, since they contribute to $F$ and thus change the radiative decay rate $\Gamma_{\mathrm{r}}$. (Also note that in practice the integration over $k_{\|}$is only carried out up to a finite cutoff value, where the contribution of surface plasmons has dropped to sufficiently small values (in most cases at around $4 \times 10^{7} \mathrm{~m}^{-1}$ )).

The simulated power dissipation spectrum can furthermore be used to calculate the fraction of power of the dipole that goes into different optical channels as discussed in the previous section:

$$
\tilde{P}_{\text {mode }}=q \cdot \int_{\lambda_{1}}^{\lambda_{2}} S(\lambda) \int_{k_{l}}^{k_{u}} P\left(k_{\|}, \lambda, z\right) d k_{\|} d \lambda,
$$

with suitable lower $\left(k_{l}\right)$ and upper $\left(k_{u}\right)$ integration limits for each channel. For this purpose the spectrum shown in Fig. 8 has to be subdivided into different regions according to the allowed range of the in-plane wave vector of the radiation to be able to couple to distinct optical modes. E.g. for direct emission into air $\left(\tilde{P}_{\text {air }}\right)$ the in-plane wave 
vector must satisfy the condition $0 \leq k_{\|} \leq\left(n_{\text {air }} \cdot k_{0}\right)$ with $k_{0}=2 \pi / \lambda$ being the vacuum wave vector at a given wavelength $\lambda$. In addition, in this case also transmission losses are included in the calculation. Finally, by dividing the power contribution of the air mode by the total power dissipation one arrives at:

$$
q_{\mathrm{eff}} \cdot \eta_{\mathrm{out}}=\frac{\tilde{P}_{\mathrm{air}}}{\tilde{P}_{\mathrm{tot}}},
$$

from which the outcoupling factor (for direct emission to air) is easily obtained, if $q_{\text {eff }}$ is known from Eq. 8 .

As an example, Fig. 6 shows a pie chart of the different fractions of power to which the emitting molecules in a prototypical OLED dissipate their excitation energy. Obviously, the afore mentioned $1 /\left(2 n^{2}\right)$ estimation based on ray optics is not that far from the calculated value of about $15 \%$ for the air mode. However, this is due to the fact that the distance of the emitter to the cathode was chosen not too far from the values favorable for light outcoupling (see also Fig. 12). As will be shown below, this number can vary strongly if the spacing between the emitter and a metallic electrode is varied. It is further evident from this chart that the amount of light that is accessible directly and via substrate light extraction is limited to about $40 \%$ in this particular OLED stack. The biggest loss is the coupling to surface plasmons, amounting to more than $40 \%$ of the total power.

In order to verify simulation results with experimental data one can on the one hand use measured EQE values (without and with a macro-extractor attached to the glass substrate), which is an angle integrated quantity. However, as expressed in Eq. $1 \eta_{\mathrm{EQE}}$ is a product of four factors so that additional assumptions are necessary. On the other hand one can also use angular and polarization dependent emission spectra containing much more information. Figs. 9 and 10 show a comparison of measured and simulated spectra where all observed features (in particular the appearance of an s-polarized substrate mode) are well reproduced. Moreover, a detailed analysis of the angular emission spectra allows to recalibrate the layer thicknesses of the OLED stack (as shown in Fig. 10) and to determine the spatial extend of the emission profile as well as the orientational distribution of the transition dipole moments being particularly important in polymer OLEDs $[17,57,59$, $60]$.

4.3 Simulation-based Optimization of OLED Layer Stacks The real benefit of optical simulations is to study and optimize OLED stacks without the need for elaborate and time-consuming experiments. In this section, the prototypical $\mathrm{Alq}_{3}$ OLED introduced in Sec. 2 will be investigated in terms of thickness variations of the electron transport layer (ETL) and hole transport layer (HTL). This basically corresponds to a variation of the distance between the emitter position and the interfaces to the metallic electrode and the glass substrate. Thus, the cavity will be strongly modified, which results in changes of the
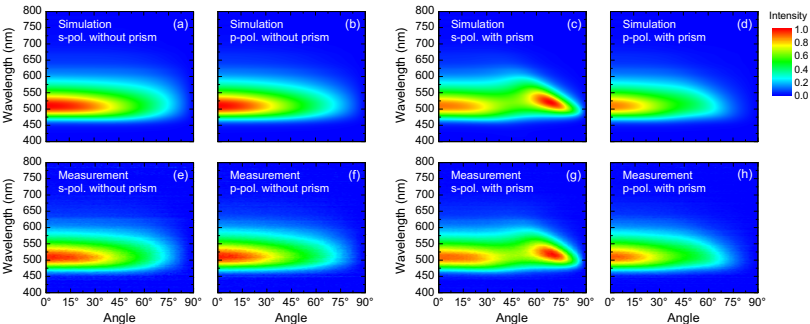

Figure 9 Simulation (upper row, a-d) and measurement (lower row, e-h) of the spectrally resolved angular dependent emission of the $\mathrm{Alq}_{3}$ OLED in the case of s- and p-polarized light. Left half is data for the planar OLED, right half shows the results for an index-matched fused silica half-cylinder prism attached as a macro-extractor to the glass substrate. (See also Ref. [40].)
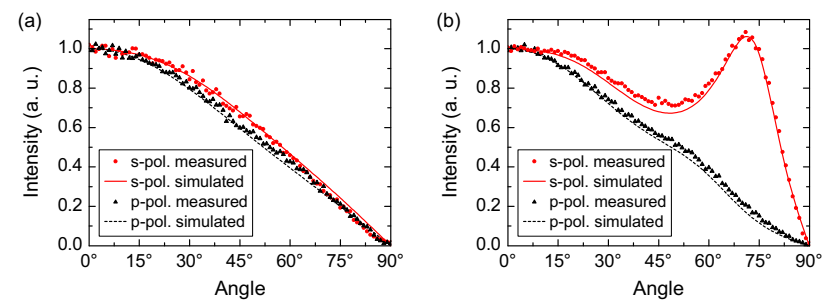

Figure 10 Cross section at a wavelength of $510 \mathrm{~nm}$ of the sand p-polarized angular dependent measurement and simulation of Fig. 9. (a) Direct emission to air, (b) emission with attached macro-extractor. Best agreement is obtained with layer thicknesses of $60 \mathrm{~nm}$ for $\mathrm{Alq}_{3}$ and $77 \mathrm{~nm}$ for S-TAD as given in Fig. 4.

outcoupling efficiency into air and into the substrate. We will assume a radiative quantum efficiency of $q=1$ in this example. Thus, variations of the cavity environment will only affect the light outcoupling factor $\eta_{\text {out }}$; the effect on $q$ will be discussed separately in the next section.

Fig. 11 shows how the fraction of power that is dissipated into different optical modes varies as a function of the ETL thickness in the prototypical $\mathrm{Alq}_{3}$ OLED. Oscillations are clearly observable for direct emission into air and substrate emission. As the distance increases, the strength of the oscillations decreases because the intensity of the dipole radiation field weakens with increasing distance from the dipole, thus the strength of the reflected field will also be decreased [55]. It is noteworthy that the optimum for extraction to air is not in the first cavity maximum at about $75 \mathrm{~nm}$ ETL thickness but in the second antinode at about $220 \mathrm{~nm}$ (in agreement with similar results by Lin et al. [61]). However, if the contribution of both the direct and the substrate emission are considered, i.e. if substrate light extraction enhancement tools are used, then the optimum ETL thickness will be at about $90 \mathrm{~nm}$.

Power dissipation to waveguide modes and surface plasmons shows a quite different progression. Coupling to waveguides is very low for small ETL thicknesses, but it is strongly enhanced with increasing thickness. This 


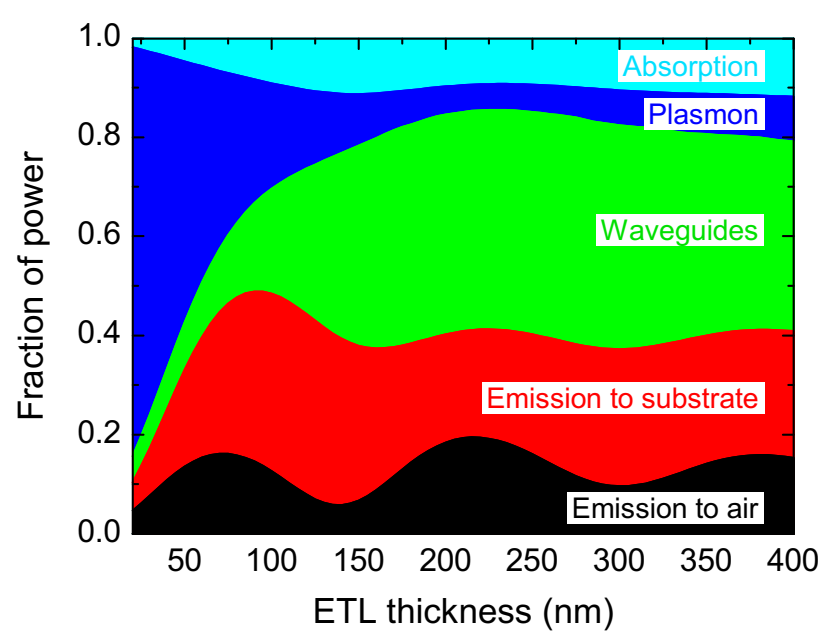

Figure 11 Amount of power coupled to different optical channels for the $\mathrm{Alq}_{3}$ OLED as depicted in Fig. 4 in dependence of the electron transport layer $\left(\mathrm{Alq}_{3}\right)$ thickness assuming a radiative quantum efficiency of $q=1$. The simulation was performed polychromatically according to the emission spectrum of $\mathrm{Alq}_{3}$. (See also Ref. [40].)
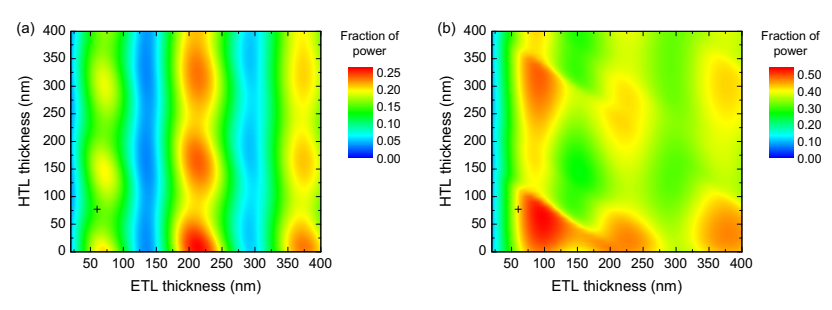

Figure 12 Optical power coupled directly into air (a) and into the glass substrate for an applied macro-extractor (b) in dependence of the electron and hole transport layer thicknesses. The stack consists of a glass substrate, $140 \mathrm{~nm}$ ITO, $30 \mathrm{~nm}$ PEDOT:PSS, varying thicknesses of S-TAD (HTL) and $\mathrm{Alq}_{3}$ (ETL), $10 \mathrm{~nm} \mathrm{Ca}$ and $100 \mathrm{~nm} \mathrm{Al}$. The position of the prototypical OLED stack used in this work (cf. Fig. 4) is marked by a cross in the diagram. The simulations were performed at a single wavelength of $510 \mathrm{~nm}$, i.e. close to the emission maximum of $\mathrm{Alq}_{3}$. The ETL and HTL thicknesses were varied in steps of $5 \mathrm{~nm}$ and $10 \mathrm{~nm}$, respectively. A radiative quantum efficiency of $q=1$ was assumed.

can be explained by the fact that there is a lower limit for the thickness of an asymmetric waveguide before a waveguide mode can exist. Above this limit an increasing number of modes are supported for larger thicknesses. By contrast, power dissipation to SPPs is strongest for a small ETL thickness, i.e. if the emitter is positioned close to the metallic cathode. This is obvious, because SPPs are evanescent waves which are only excited if the dipole couples to the metal in the near-field. This contribution is reduced with increasing distance of the dipole to the metal. It should be noted that the simulation does not differentiate between coupling to surface plasmons and non-radiative

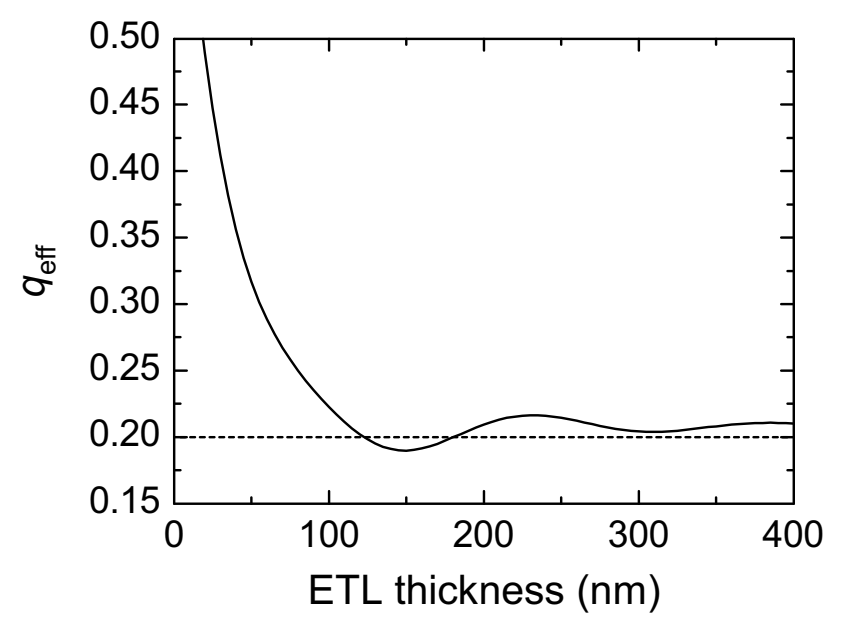

Figure 13 Variation of $q_{\text {eff }}$ with the distance of the emitting dipoles to the cathode in the prototypical OLED given in Fig. 4. The emission layer is assumed to be an infinitely thin sheet of $\mathrm{Alq}_{3}$ molecules with $q=0.2$ residing immediately at the $\mathrm{Alq}_{3} / \mathrm{S}$ TAD interface. (See also Ref. [40].)

energy transfer to lossy surface waves, i.e. luminescence quenching at the metal, which especially dominates for very small distances below about $25 \mathrm{~nm}$ and significantly reduces the efficiency $[41,55,62]$.

Further optimization of the OLED layer stack is possible if both the ETL and HTL are systematically varied in thickness as shown in Fig. 12. For the sake of simplicity, these simulations have been carried out at a single wavelength of $510 \mathrm{~nm}$ only (in the emission maximum of $\mathrm{Alq}_{3}$ ). It is again found that changing the ETL thickness has the strongest impact on EQE as the distance to the cathode is the main determining factor for interference effects. Moreover, reducing the HTL thickness (or in practice the ITO thickness) will lead to a further improvement of the efficiency [63].

As a result, modifying the cavity environment of the emitting molecules allows for optimizing the light outcoupling of an OLED. Nevertheless, coupling to both surface plasmons and waveguide modes causes combined losses of around $50 \%$ of the total available power - almost independent of the chosen thickness. Therefore, it is inevitable to either reduce coupling to these unfavorable loss channels or at least to partially recover some of the dissipated energy in order to develop highly efficient OLEDs. For this reason, we will later on present several promising approaches.

\subsection{Influence of the Emitter Quantum Efficiency}

In the discussion so far, we have assumed that the intrinsic radiative quantum efficiency $q$ of the emitter material is equal to one. In real OLEDs, however, and in particular in the considered prototypical $\mathrm{Alq}_{3}$ OLED, this quantity is often significantly less than unity. According to the above said the cavity environment will then not only modify the light outcoupling factor $\eta_{\text {out }}$ but also the overall decay rate and thus lead to an effective radiative quantum 


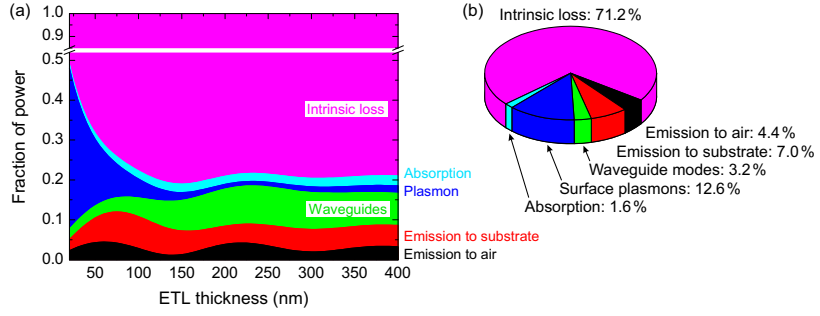

Figure 14 Amount of power coupled to different optical channels as in Fig. 11 in dependence of the electron transport layer $\left(\mathrm{Alq}_{3}\right)$ thickness assuming a radiative quantum efficiency of $q=0.2$. Also shown is a pie-chart of the different fractions of power for an $\mathrm{Alq}_{3}$ thickness of $60 \mathrm{~nm}$. (See also Ref. [46].)

efficiency $q_{\text {eff }}$ (see Eq. 8). Figure 13 shows the effect on $q_{\text {eff }}$ of the distance of $\mathrm{Alq}_{3}$ emitter molecules with $q=0.2$ to the cathode in the prototypical OLED given in Fig. 4 (cf. Ref. [40]). It is found that $q_{\text {eff }}$ oscillates around the value of $q$ and reaches the true value only asymptotically for very large distances. For small distances (less than about $120 \mathrm{~nm}$ ), however, $q_{\text {eff }}$ strongly increases due to constructive interference but most importantly due to coupling to SPPs at the metal-organic interface. We note that for distances less than about $25 \mathrm{~nm}$ the radiative quantum efficiency should strongly decrease again due to non-radiative energy transfer to the metal and subsequent quenching of the excitation $[52,55,62]$, which is not distinguished for SPPs in our analysis.

The consequences for the efficiency of OLEDs with emitters having $q \ll 1$ are manifest. Due to the enhancement of $q_{\text {eff }}$ for smaller distances to the metal cathode, the first cavity maximum will gain in power relative to the second one, where the cavity effect on the radiative rate is much weaker. This is directly observable in Fig. 14, where the dissipation of power to different optical channels is shown for the same prototypical $\mathrm{Alq}_{3}$ OLED stack as before (Fig. 11), but now with a realistic value of $q=0.2$ [46]. As expected, the biggest loss is now due to intrinsic non-radiative exciton decay, however, for both direct emission to air and emission to substrate the optimum thickness is at the first cavity maximum below $100 \mathrm{~nm}$ distance to the cathode. And it is also worth noting that the fraction of power which can be coupled out of the device is significantly more than what would be expected by taking the simulation for $q=1$ and simply multiplying with the lower $q$-value 0.2 . Thus the correct value of $q_{\text {eff }}$ is essential for making reliable OLED efficiency predictions according to Eq. 1.

\subsection{Comprehensive Efficiency Analysis of OLEDs} As outlined in the previous section, the knowledge of the intrinsic radiative quantum efficiency is of paramount importance for OLED optimization, in particular for designing the optimum layer stack. In many cases, however, this information is not available or the value of $q$ is taken from photoluminescence measurements performed on films fab-
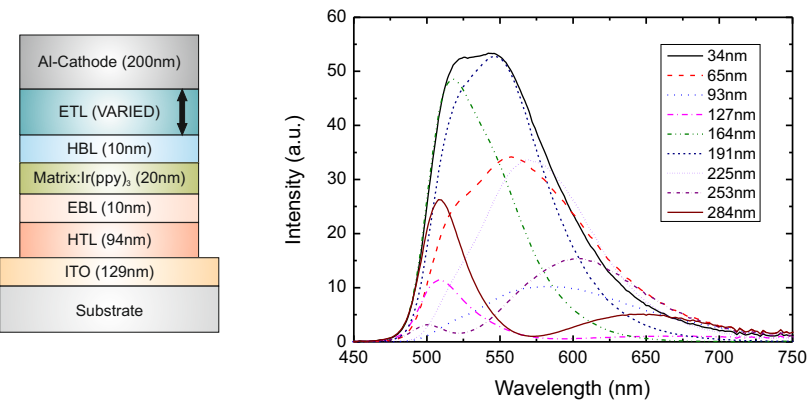

Figure 15 Green phosphorescent OLED stack with $\operatorname{Ir}(\mathrm{ppy})_{3}$ as emitter, where the ETL thickness is systematically varied. Simulated emission spectra show both spectral as well as intensity changes, indicating strong effects of the variation of the optical cavity, while the electrical characteristics (not shown) are not affected. (See also Ref. [65]).

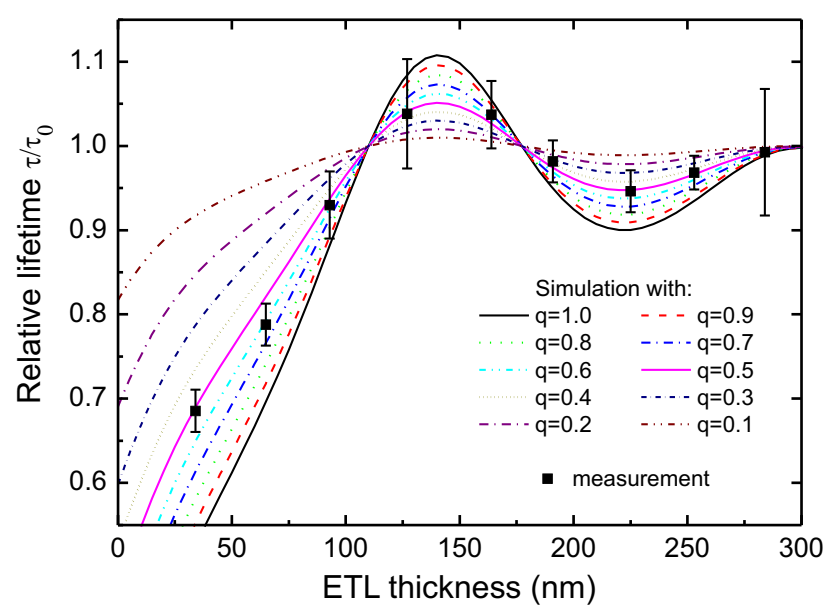

Figure 16 Experimentally determined phosphorescence lifetimes of $\operatorname{Ir}(\mathrm{ppy})_{3}$ in OLED stacks with different ETL thickness as shown in Fig. 15. The lines are simulations for the given stacks with different values of the intrinsic radiative quantum efficiency q. (See also Ref. [65]).

ricated under quite different conditions than an OLED. Furthermore, in an operating OLED the excitation profile is usually different than in an optical experiment and excitons can be quenched at interfaces or due to interactions with other excited molecules or charge carriers residing in the emission layer. Thus, a method for the determination of $q$ applicable directly in an OLED environment or even in an operating OLED will give the most direct information on the value of the radiative quantum efficiency [64]. We have recently shown that the above described variation of the distance between the emitter and a metal electrode, which changes the optical environment most significantly, can be performed in real OLEDs without changing the electrical properties, provided that a doped electron transport layer is used $[39,65]$. 


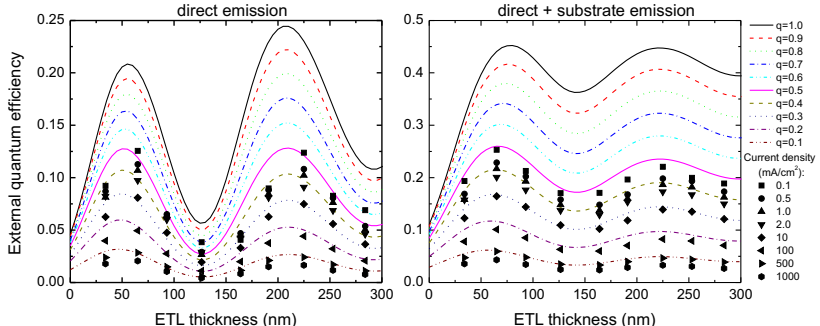

Figure 17 Experimentally determined external quantum efficiencies of OLED stacks with different ETL thicknesses as shown in Fig. 15 (left: direct emission to air; right: values obtained with a macro-extractor). The lines are simulations for the given stacks with different values of the intrinsic radiative quantum efficiency q. (See also Ref. [65]).

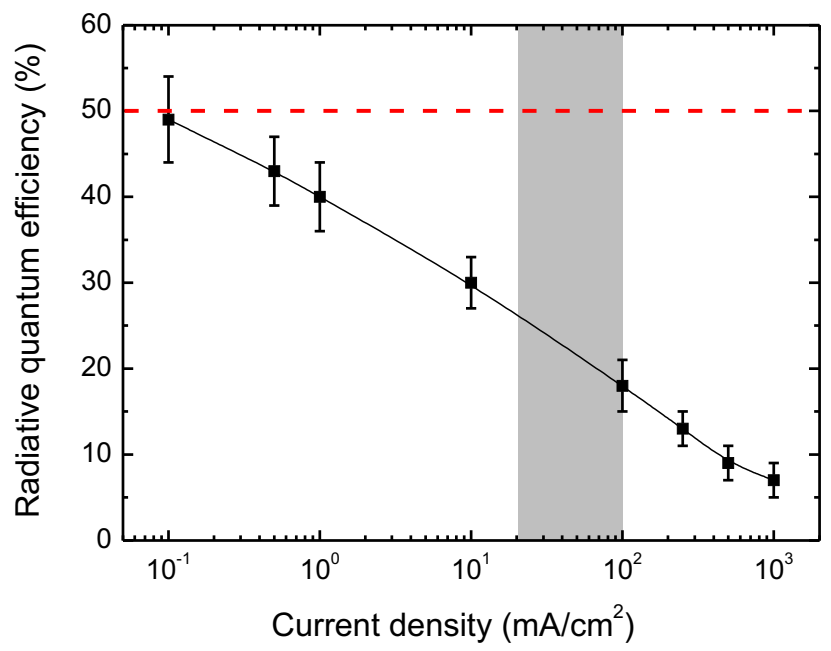

Figure 18 Dependence of the extracted radiative quantum efficiency from Fig. 17 as a function of the driving current of the OLEDs. The horizontal dashed line corresponds to a $q$-value of 0.5 determined from photoluminescence measurements; the vertical shaded area indicates the typical drive conditions for OLEDs in lighting applications. (See also Ref. [65]).

In order to prove the feasibility of this approach, a green phosphorescent OLED based on $\operatorname{Ir}(\mathrm{ppy})_{3}$ as emitter was chosen (see Fig. 15, for details we refer to Ref. [65]). Photoluminescence lifetime measurements were performed on a variety of OLED stacks with different ETL thickness. As shown in Fig. 16, the phosphorescence lifetime of $\operatorname{Ir}(\text { ppy })_{3}$ is not constant but varies with the ETL thickness. This directly reflects the influence of the optical cavity on the radiative decay rate of the emitter. With the above presented optical modeling a quantitative analysis is now possible. Recall that the measured PL lifetime $\tau$ in the presence of a cavity is given by $\tau=\Gamma^{-1}=\left(\Gamma_{\mathrm{r}}^{*}+\Gamma_{\mathrm{nr}}\right)^{-1}$ and in the absence of the cavity by $\tau_{0}=\Gamma_{0}^{-1}=\left(\Gamma_{\mathrm{r}}+\Gamma_{\mathrm{nr}}\right)^{-1}$. Thus, according to Eq. 7 the ratio between these two values only depends on the cavity enhancement factor $F$ and the intrinsic radiative quantum efficiency of the emitter $q$, but is independent of the light outcoupling factor:

$$
\frac{\tau}{\tau_{0}}=\frac{\Gamma_{0}}{\Gamma}=[(1-q)+q \cdot F]^{-1}
$$

As $F$ is obtained independently from simulation for each of the used OLED stacks with different ETL thicknesses, the two free parameters $q$ and $\tau_{0}$ are easily determined by comparing measured lifetime data with simulation results. As shown in Fig. 16, the best agreement is obtained with $q_{(P L)}=0.5 \pm 0.1$ and an intrinsic phosphorescence lifetime $\tau_{0} \approx 700$ ns. Remarkably, the value of the quantum efficiency is significantly less than unity. Thus, care should be taken when published IQE values for the emitter $\operatorname{Ir}(\mathrm{ppy})_{3}$ [66] are generalized to other OLED stacks containing this emitter. $q$ should rather be considered as an OLED stack specific quantity, depending, e.g., on the type of matrix material for the emitting dye or the materials in the immediate vicinity of the emission layer. Other factors could be concentration quenching and/or the effect of dye aggregation.

The same series of OLEDs with systematic variation of the ETL thickness can now be used for the determination of the radiative quantum efficiency $q_{(E L)}$ under electrical operation, which need not necessarily be identical to $q_{(P L)}$ as will be seen below. For this purpose the EQE of the complete series of OLEDs was measured in an integrating sphere, once without any substrate modification and once with an index-matched hemispherical lens attached to the glass substrate to extract all of the substrate modes. Both measurements were performed for various current densities to investigate possible effects of exciton quenching with increasing currents. Again, the experimental results can nicely be reproduced by optical simulation as shown in Fig. 17 with $q$ being the only free parameter. As demonstrated in Ref. [65], the charge balance factor $\gamma$ can thereby be assumed to be close to unity. The analysis now allows extracting the radiative quantum efficiency $q_{(E L)}$ at each current density as shown in Fig. 18. It is evident that only in the limit of very small currents $q_{(P L)}$ and $q_{(E L)}$ take roughly the same value of 0.5 , however, with increasing current density $q_{(E L)}$ rapidly decreases and may only be some $20 \%$ in the range where OLEDs are typically operated for lighting applications. Thus one has to conclude that for simulation based optimization of OLEDs, i.e. for example the question whether the emitter should be placed in the first or second cavity maximum, not only has the radiative quantum efficiency $q$ in the limit of low excitation densities to be known, but also its value under realistic operating conditions.

Finally, a comprehensive efficiency analysis of OLEDs has to include the investigation of the radiation pattern, i.e. the angular and polarization dependent emission spectra as mentioned before, to obtain information about the emitter orientation $[59,60]$ and the spatial extend of the 


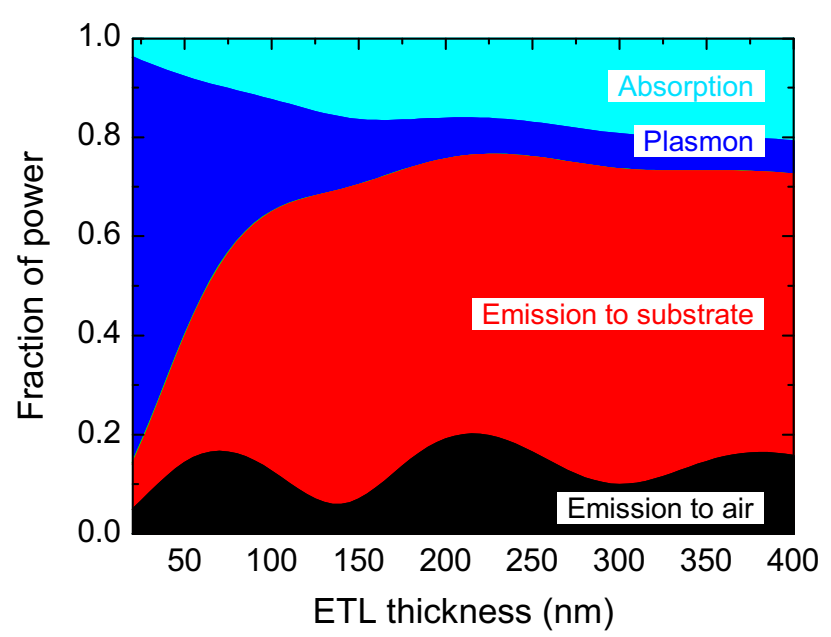

Figure 19 Amount of power coupled to different optical channels in dependence of the electron transport layer thickness for the $\mathrm{Alq}_{3}$ OLED as depicted in Fig. 4, but now with a high-index substrate (SF6 glass with $n=1.82$ ). The simulation was performed polychromatically according to the emission spectrum of $\mathrm{Alq}_{3}$, and a radiative quantum efficiency of $q=1$ was assumed.

emission zone $[17,57]$. We have performed this analysis for the series of $\operatorname{Ir}(\mathrm{ppy})_{3}$ OLEDs and found no evidence for a non-isotropic emitter orientation of $\operatorname{Ir}(\mathrm{ppy})_{3}$ as well as good agreement with the assumption of a sharply localized emission zone in the middle of the only $10 \mathrm{~nm}$ wide EML [65]. We will later on come back to the issue of non-isotropic emitter orientation when rod-like dyes (e.g. BDASBi [67]) or asymmetrically substituted metalorganic complexes (e.g. $\operatorname{Ir}(\mathrm{MDQ})_{2}$ (acac) $[58,68]$ ) are used as emitters.

\section{Approaches to Improved Light Outcoupling}

5.1 Overview of Different Techniques As shown above, in planar bottom-emitting OLEDs typically around $50 \%$ of the generated light is trapped in waveguided and surface plasmon modes and only a fraction of about $20 \%$ of it is directly radiated into air. In the previous section we have already made use of the fact that light captured in the glass substrate can be fully extracted if an index-matched macroscopic lens is used and if the active pixel area is not too large. However, if the unique form factor (thin and flat) is to be preserved, this method is not practicable in large area devices. Thus, the development of new concepts for improving light extraction efficiency has been a major issue over recent years (for reviews see e.g. Refs. [47,69]).

The different approaches can roughly be distinguished into techniques staying with planar structures and others utilizing scattering methods [70,71]. Among the former ones are approaches towards further optimization of the OLED cavity, i.e. modifications of the layer stack with respect to thicknesses, refractive indices or reflectivity. A relatively simple method is to place the emission zone in the second antinode of the interference pattern relative to the cathode [61] and thus reduce the coupling to SPPs. As discussed before, however, this will only be beneficial if the radiative quantum efficiency of the emitter is very high; otherwise the emitter will experience a stronger enhancement of its radiative rate when placed in the first cavity maximum. Alternatively, the excitation of SPPs can be completely avoided if metal-free OLEDs are used [72], but there is usually no overall gain in light outcoupling since the reduction of SPP losses is mostly at the expense of enhanced waveguiding [46].

Another way of boosting the direct emission to air is the use of microcavity structures, i.e. OLEDs with two (partially) reflecting electrodes, where the radiation is directed more towards small angles with respect to the surface normal. This can be achieved, e.g., by (aperiodic) dielectric Bragg reflectors placed underneath the ITO electrode [73] or in top-emitting devices with a highly reflective anode on glass and a semitransparent metallic cathode, often followed by a dielectric capping layer as antireflection coating [74-76]. Though very high EQE values close to $30 \%$ have recently been published for a red phosphorescent top-emitting OLED [77], these structures usually have a non-Lambertian emission characteristics with non-negligible spectral shifts as a function of the viewing angle. We also want to note that in case of the particular red phosphorescent dye $\operatorname{Ir}(\mathrm{MDQ})_{2}$ (acac) used in Ref. [77] nonisotropic emitter orientation might at least partially contribute to the high EQE values as will be discussed at the end of this article $[58,68]$.

A third method where the planar layer structure is preserved lies in matching the refractive indices of the emission layer, where the light is generated, and the outside world, either by bringing the refractive index of the EML down to one or by using a high-index (HI) substrate. Simulations show that if the refractive index of the EML could be reduced to $n=1$, a theoretical limit of almost $70 \%$ direct emission to air would be possible [78]. An experimental realization might be difficult simply because no such materials are available, but lowering $n_{\mathrm{EML}}$ below the value of glass already would bring an enormous boost in EQE.

The use of glass substrates with higher refractive index than the organic layers (including ITO) is possible $[79,80]$. Simulations for the prototypical $\mathrm{Alq}_{3}$ OLED demonstrate (see Fig. 19) that in this way all the light from waveguided modes goes into the HI glass substrate from where it can be extracted more easily. Combining HI substrates with a macro-extractor (an index-matched lens), record EQEs in excess of $40 \%$ have been achieved in this way [20,81]. Nevertheless, one has to be aware that HI glass substrates would increase the overall cost of OLEDs considerably. Thus for practicable devices thin-film solutions have to be applied.

A fundamentally different approach to extract trapped light in OLEDs is the use of scattering structures. Here one has to distinguish between periodic and non-periodic (ran- 

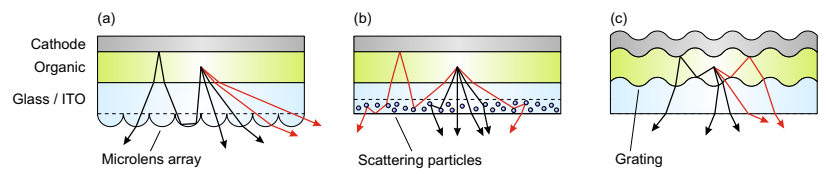

Figure 20 (a) Schematic illustration of substrate mode extraction by a microlens array. Light paths indicated in red correspond to radiation that would normally not be able to leave the device due to total internal reflection at the glass/air interface. Multiple reflections inside the stack reduce the efficiency due to absorption in the organic layers and in ITO as well as due to reflection losses at the cathode. (b) Concept of light outcoupling by scattering particles in a film applied to the backside of the substrate. (c) Realization of an internal scattering structure by a periodic grating placed between the glass substrate and the thin film stack.

dom) structures on the one hand and internal or external scattering on the other hand (see Fig. 20). The latter realization is quite straight-forward and can be any modification of the backside of the glass substrate that serves to scatter out light rays that would otherwise suffer from total internal reflection at the glass/air interface. Examples are ordered micro-lens arrays [82,83], scattering particles [84] or mechanical roughening of the glass substrate [85]. In the meantime light scattering foils are commercially available, but in contrast to a macro-extractor these techniques typically extract only part of the light trapped in the substrate and the reflecting appearance of the OLED in the off-state changes to a milky, non-reflecting one.

If one wants to get access to waveguided modes, an internal scattering structure, e.g. placed between the glass substrate and the ITO layer, has to be used. Thereby both periodic gratings (photonic crystals) or random scattering structures are possible. The effectiveness of this approach relies on the spatial overlap of the waveguide modes with such features; in other words, they have to be employed close to the emission zone of the OLED [86]. In general, photonic crystal structures with periodicities on a length scale comparable to optical wavelengths have the disadvantages that they require elaborate fabrication techniques and that they induce a wavelength and angular dependent scattering efficiency. Other interesting approaches are therefore the use of so-called low-index grids with periods on the micron scale [87] or random structures fabricated by morphological instabilities of thin film structures [88]. The above said is also true for the extraction of surface plasmons by scattering structures which consequently need to be in the vicinity of the metal cathode [89]. This boundary condition is even more challenging since many of the established patterning techniques are not compatible with OLED technology as they would damage or destroy the underlying organic films.

\subsection{Reduction of Surface Plasmon Losses}

5.2.1 Basic Properties of SPPs Before presenting our own results on the reduction of surface plasmon losses some general properties of SPPs will be discussed (for de-

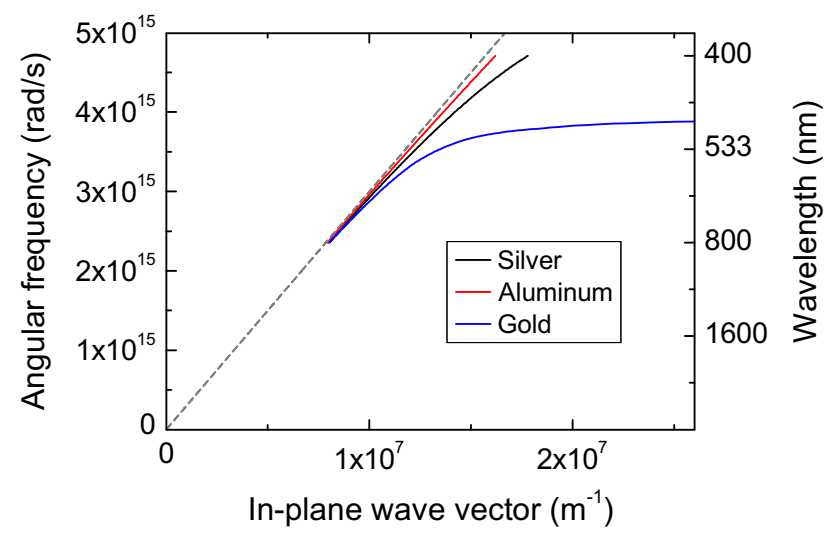

Figure 21 Dispersion relation of surface plasmon polaritons at metal/air interfaces for three different metals in the visible spectral range. The dashed line is the light-line in air following the relation $\omega=c \cdot k_{\|}$.

tails we refer to Refs. [90-92]). Surface plasmon polaritons are longitudinal, p-polarized waves traveling at the interface between a metal and a dielectric with evanescent fields decaying exponentially into both adjacent media. For semi-infinite layers their dispersion relation is given by:

$$
k_{\mathrm{SPP}}(\omega)=k_{0} \sqrt{\frac{\epsilon_{1} \cdot \epsilon_{2}}{\epsilon_{1}+\epsilon_{2}}}=\frac{\omega}{c} \sqrt{\frac{\epsilon_{1}(\omega) \cdot \epsilon_{2}(\omega)}{\epsilon_{1}(\omega)+\epsilon_{2}(\omega)}}
$$

with $\epsilon_{1,2}(\omega)$ being the complex dielectric functions of the metal and the adjacent dielectric layer, respectively, and $k_{0}$ the vacuum wave vector. This relation is shown in Fig. 21 for three different metal surfaces adjacent to air as dielectric for the range of frequencies and in-plane wave vectors relevant in this context. It is obvious that the SPP dispersion curve and the light-line in air do not intersect for finite frequencies, thus energy and momentum conservation can not be fulfilled simultaneously and as a consequence SPPs can not couple to far-field radiation. The application to OLEDs, however, requires an important modification due to the fact that the involved layers are not thick enough to be treated as bulk material. Thus, the evanescent field of the SPP can extend through an adjacent thin organic layer and sense an effective refractive index and, if the metal is thin enough, SPPs can exist on both sides of the metal layer and couple with each other [93].

5.2.2 Scattering Approaches There are well established methods how SPPs can be excited by far-field radiation, most importantly by grating coupling or prism coupling [90,91]. Making use of reciprocity in optics it is quite straightforward to transfer these techniques to lightemitting structures $[89,96]$. The first approach uses a periodic grating with period $\lambda_{g}$ to scatter SPPs so that they gain an extra momentum $k_{\mathrm{SPP}}^{\prime}=k_{\mathrm{SPP}} \pm m \cdot\left(2 \pi / \lambda_{g}\right)$ (with an integer number $m$, see Fig. 22). This scattering approach relies on the fact that there is sufficient overlap between the SPP mode and a periodic modulation of the refractive 

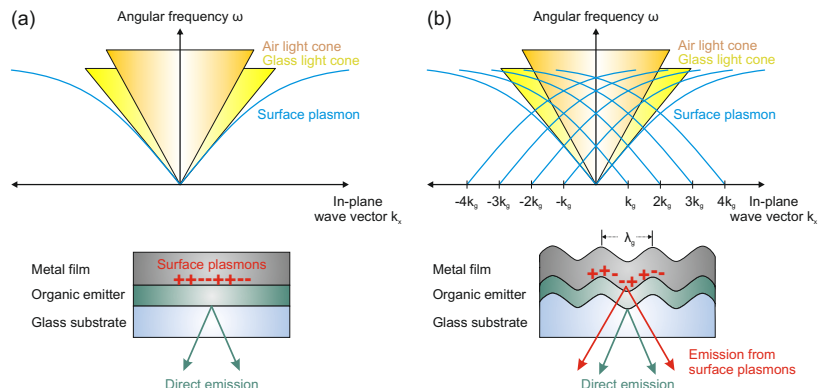

Figure 22 Simplified layer structure to demonstrate the principle of SPP grating coupling. The SPP dispersion relation, lying below the glass light-cone in planar layered structures (a), is scattered by multiples of the grating wave vector to result in several branches being located within the glass light-cone (b), so that light from SPPs can be coupled out. (See also Ref. [94]).
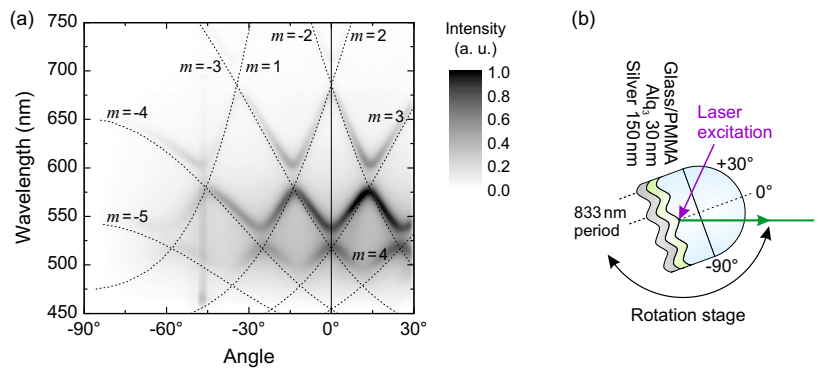

Figure 23 (a) Experimentally measured angular dependent ppolarized emission spectra after photoluminescence excitation of a $30 \mathrm{~nm}$ thick $\mathrm{Alq}_{3}$ film adjacent to a silver layer, both deposited on an $833 \mathrm{~nm}$ periodic line grating that was nanoimprinted into a PMMA layer. The dotted lines show simulated SPP dispersions of the corresponding planar structure, where the curves were shifted by integer multiples of the grating wave vector. (b) Schematic sample layout used in the experiment; both excitation and detection are through the glass prism. Excitation is made by a $375 \mathrm{~nm}$ laser diode incident at a fixed angle of $45^{\circ}$, detection is performed by a fibre optical spectrometer through a linear polarizer in front of which the sample is rotated on a motorized turn table. (See also Ref. [95]).

index, which implies that the grating has to be placed next to the metal layer (or there has to be a height modulation reaching through the complete layer stack including the cathode). The fabrication of OLEDs fulfilling this condition is still challenging [97], as not only the optical properties have to be tuned but also the electrical functioning has to be ensured. For that reason we have investigated this coupling approach only in simplified structures comprising a luminescent film adjacent to a metal layer $[94,95]$.

Fig. 23 shows experimentally measured angular dependent p-polarized emission spectra for photoluminescence excitation of a $30 \mathrm{~nm}$ thick $\mathrm{Alq}_{3}$ film adjacent to a silver layer, both deposited on a line grating that was nanoimprinted into a PMMA layer. One can clearly see that SPPs,

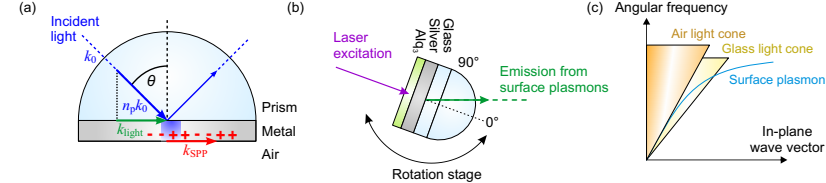

Figure 24 Principle of SPP index coupling: (a) shows the excitation of SPPs by light from the far field in the so-called Kretschmann configuration. (b) Inverse Kretschmann configuration to extract SPPs that are excited by near-field coupling from a luminescent organic film. (c) The dispersion relation of the SPP travelling at the interface to air overlaps with the light-cone for the attached glass prism. (See also Ref. [94]).
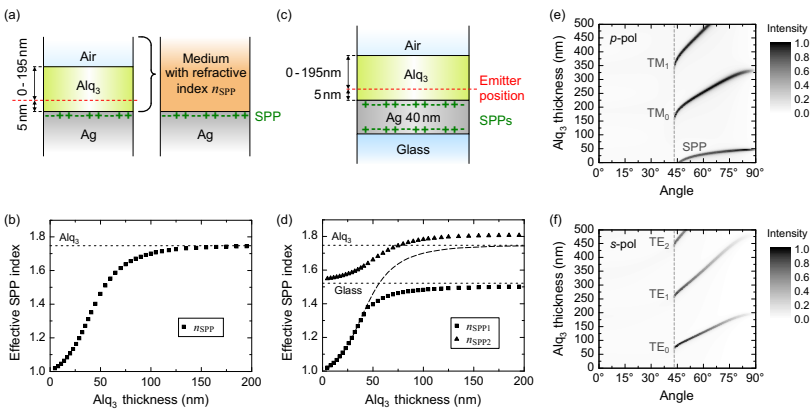

Figure 25 Concept of the effective refractive index of the adjacent dielectric medium that is probed by an SPP for a thick metal layer (a, b) and for a thin metal layer (c, d). Also shown is the simulated angular dispersion of the modes that can be extracted through a fused silica prism as a function of the organic layer thickness: (e) p-polarized, (f) s-polarized. (See also Refs. [94, 99]).

which are excited by fluorescent $\mathrm{Alq}_{3}$ molecules via nearfield coupling, are scattered back into the substrate up to the fifth diffraction order. There is excellent agreement between the measured angular dispersion and the simulated curves using the above described methods. One can also readily show that waveguided modes which will become relevant for thicker organic layers are equally well scattered out in this way. Furthermore, we have demonstrated that even structures containing some randomness, produced e.g. by an ordinary DVD stamp, can be used [95]. Nevertheless, one has to be aware that periodic structures will induce a pronounced angular dependence of the perceived colour of the OLED [98]. Thus, for white OLEDs with colour coordinates independent of the viewing angle random scattering structures have to be used [88].

5.2.3 Index Coupling Fig. 24 shows the principle of index (or prism) coupling utilizing the so-called inverse Kretschmann configuration [100]. This technique makes use of the fact that the in-plane wave vector within the prism is stretched by a factor given by the refractive index of the prism, so that energy and momentum conservation are fulfilled. If the refractive indices are properly chosen, it is thus possible to extract SPPs that are evanes- 

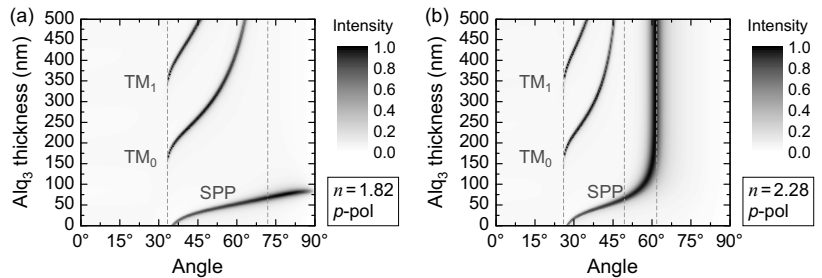

Figure 26 Simulated angular dispersion of the p-polarized modes extracted through a high index SF6 glass prism (a) and a $\mathrm{LiNbO}_{3}$ prism (b) as a function of the organic layer thickness. (See also Ref. [99]).

cent modes at the metal/organic interface (the bottom-side in Fig. 24) to become radiative modes at the opposite side of the metal layer (here the top-side). To realize this concept, however, some prerequisites have to be fulfilled. First, the metal film obviously has to be semitransparent. Since there are two counteracting processes, namely an enhanced coupling of the emitting dipoles to SPPs with an increasing metal thickness on the one hand and a concomitant reduced optical transmission on the other hand, there is typically an optimum thickness at around $50 \mathrm{~nm}[94,101]$. More importantly, the refractive index on the extraction side of the metal has to be larger than on the other side in order to match the dispersion relation of the plasmon (traveling at the bottom side) with the far-field light-line in the prism at the top side. Due to the evanescent nature of SPPs this condition not only depends on the bulk values of the organic material and the prism, but in particular on the thickness of the organic layer. Since the decay length of the SPP field amplitude perpendicular to the metal/dielectric interface is typically of the order of half a wavelength, the SPP field probes an extended vertical distance to the metal surface. In order to account for this feature we have introduced an effective refractive index that has to be entered in Eq. 13 to calculate the correct SPP dispersion relation. This concept of the effective index of SPPs and its dependence on the thickness of the organic layer for a one-sided SPP (thick metal) and a two-sided SPP configuration (thin metal) is shown in Fig. 25. Without going into the details (see e.g. Ref. [99]), it is clear from the figure that only for thin $\mathrm{Alq}_{3}$ layers (up to about $50 \mathrm{~nm}$ ) ordinary glass can be used to extract SPPs. For larger organic layer thickness, and in particular for the thicknesses typically used in OLEDs, the extraction of SPPs (in addition to waveguided modes that can also be extracted by this method) requires a medium on the top-side of the metal that has a significantly higher refractive index.

Figure 26 shows exemplarily the calculated angular dispersion of p-polarized modes that can be extracted if a high index glass (SF6, $n=1.82)$ or a $\mathrm{LiNbO}_{3}(n=2.28)$ prism are used. In the former case SPP modes can now be extracted up to an organic layer thickness of about $100 \mathrm{~nm}$, while in the latter case the index is so high that there is no limit any more and all modes, regardless of the thickness (a)
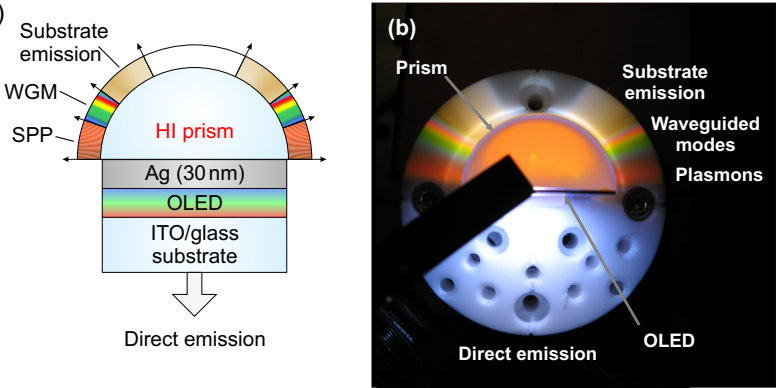

(c)
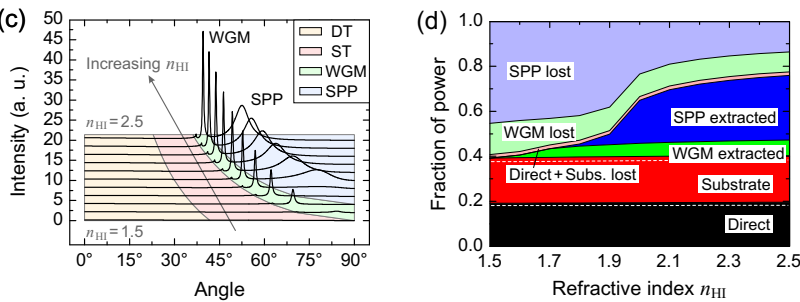

Figure 27 Sample layout (a) and photograph (b) of a white OLED with direct emission towards the bottom and extraction of all other modes to the top-side using an SF6 prism with $n=1.82$. The simulations in (c) show the angular dispersion of the modes extracted through the high-index glass prism and in (d) the angle integrated contribution of the different modes as a function of the refractive index of the prism. Note that these simulations were performed at a single wavelength of $600 \mathrm{~nm}$ and a semi-infinite glass substrate was assumed to avoid back reflected light from the glass-air interface appearing as top emission. (See also Refs. [99, 102]).

of the organic film, can be extracted under an angle of not more than $60^{\circ}$.

The application of this concept to a white bottomemitting OLED with a thin semi-transparent Ag topcontact is presented in Fig. 27. The photograph clearly shows that only the air mode is emitted to the bottom side, whereas all other modes (including substrate, waveguide and SPP modes) are emitted in characteristic angular ranges to the top side. We note that the index of the used SF6 prism was not high enough to extract the SPP completely, so that only the red part of the SPP branch is seen under large angles close to $90^{\circ}$. Furthermore, the used index matching fluid was strongly absorbing in the short wavelength range so that the substrate light has a brownish appearance. As the simulations in (c, d) show, the extraction of a major fraction of the energy contained in SPPs requires a medium with $n_{\mathrm{HI}}>2.0$. Nevertheless, this is a clear demonstration that the concept of high-index coupling is not only applicable to extract wave-guided modes in bottom-emitting OLEDs, as was demonstrated before by different authors $[20,80]$, but moreover to get access to all trapped modes - including surface plasmons [99].

It should be noted, however, that these results can only serve as a proof of principle, because using a high-index 


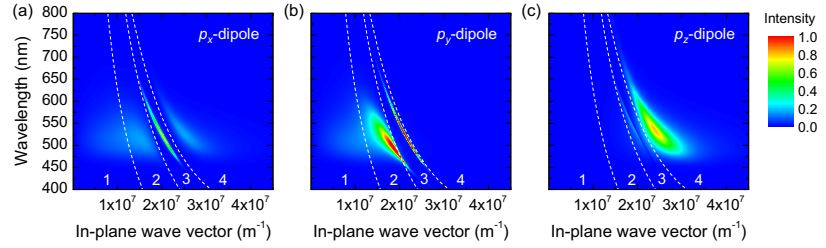

Figure 28 Simulation of power dissipation for the prototypical $\mathrm{Alq}_{3}$ OLED (structure shown in Fig. 4) for the three orthogonal dipole orientations separately $(x, y$ in the substrate plane, $z$ perpendicular to the substrate plane). In all cases the radiative quantum efficiency was set to $q=1$. The dashed lines separate the power dissipation spectra into different regions as denoted in Fig. 8. (See also Ref. [46]).

prism is not practicable in OLED applications. It would therefore be necessary to develop thin-film solutions to get rid of the viewing angle dependent colour shift. In that context it should be noted that top-emitting OLEDs with highindex capping layers have been known for many years [74, 75], however, the connection to SPPs was not made. Only recently has it been shown that including a fluorescent dye in such a capping layer can allow to extract energy from SPPs in top-emitting OLEDs [103].

5.2.4 Emitter Orientation Instead of developing tools to extract energy from surface plasmons, one can also consider means to reduce their excitation in OLEDs. Apart from the already mentioned distance dependent coupling between a radiating dipole and SPPs there is also a characteristic dependence on the orientation of the dipole $[51,55]$. Thus another way to avoid the excitation of surface plasmons, even if the emitter is rather close to the metal, is to control the orientation of the emitting molecules and thus of their transition dipole moments. Keeping the radiation pattern of a classical electrical dipole in mind (cf. Fig. 7) and considering that surface plasmons are transverse magnetic modes, one readily concludes that perfectly horizontally oriented dipoles would only very weakly couple to SPPs [45]. This effect has been known for many years in polymeric OLEDs [104], only very recently, however, we have been able to show that orientation effects also play a role in small molecule OLEDs fabricated by vacuum evaporation, where the fluorescent or phosphorescent dyes are embedded with only a few percent content in a matrix material $[58,67,68]$.

To demonstrate the potential of controlling the emitter orientation for efficiency enhancement in OLEDs, Fig. 28 shows simulated power dissipation spectra separately for each of the three orthogonal dipole orientations in the prototypical Alq $\mathrm{ALED}_{3}$ (structure shown in Fig. 4). From this plot one can clearly see that horizontal dipoles $\left(p_{x}\right.$ and $\left.p_{y}\right)$ couple to various optical channels, whereas vertical $\left(p_{z}\right)$ dipoles dissipate their energy almost exclusively to SPPs, which makes it difficult to detect them in OLEDs, e.g. from angular dependent emission spectra of OLEDs. As a workaround we have therefore developed a method which
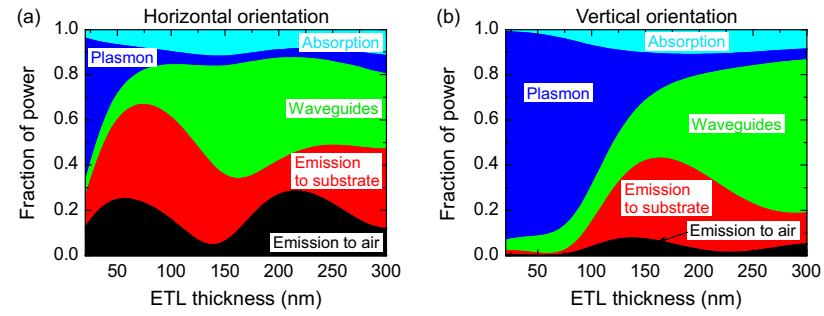

Figure 29 Simulation of power dissipation for exclusively horizontal (a) or vertical dipole orientation (b) in the prototypical $\mathrm{Alq}_{3}$ OLED (structure shown in Fig. 4) as a function of the ETL thickness. In both cases the radiative quantum efficiency was set to $q=1$. (Compare to Fig. 11 for the isotropic case.)

is based on photoluminescence excitation of layer stacks that contain the same EML as the corresponding OLED but no metal layer [105]. It is thus possible to quantitatively determine the orientational distribution from angular and polarization dependent photoluminescence spectra.

It is furthermore instructive to look at the power dissipation of both dipole orientations into different optical channels as a function of the distance to the cathode, as displayed in Fig. 29 (cf. Fig. 11 for the isotropic case). It is obvious from these simulations that the light outcoupling efficiency (be it only direct emission or including substrate emission) can be enhanced by about a factor of 1.5 with respect to the case of random emitter orientation, which is mostly due to the strongly reduced coupling to SPPs, if vertical dipoles are absent. Thus, from the point of view of efficiency, the vertical dipole orientation should be avoided in OLEDs. It is also interesting to note in this context, that vertical dipoles exhibit markedly different distance dependent coupling to the various optical channels as compared to horizontal ones, which is due to different interference conditions. First, the coupling of vertical dipoles to SPPs has a longer range than in the horizontal case (as predicted already in Ref. [106] for energy transfer of dyes to a metal surface). Second, for vertical dipoles the maximum outcoupling efficiency to air is achieved for an ETL thickness of about $135 \mathrm{~nm}$, exactly where the outcoupling from horizontal ones has an interference node. This property can be used for the determination of the amount of vertical dipoles in an OLED with non-isotropic emitter orientation by intentionally fabricating a non-optimized layer stack [60].

To realize this concept one needs emitter molecules that show non-isotropic orientation in a small molecule OLED environment, where the emission layer is usually a thin dye-doped layer prepared by co-evaporation on top of other - usually amorphous - layers. Good candidates are rod-like chromophores with a large shape anisotropy, such as the blue fluorescent dye BDASBi shown in Fig. 3. This material has already been known to exhibit non-isotropic orientation in neat evaporated films [107], but interestingly it preserves this feature even when it is doped into a CBP matrix [105]. 
(a)

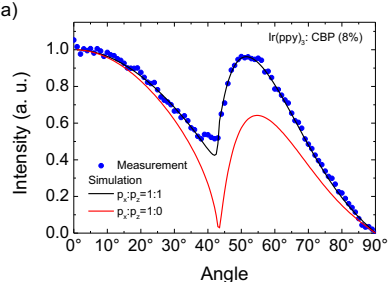

(b)

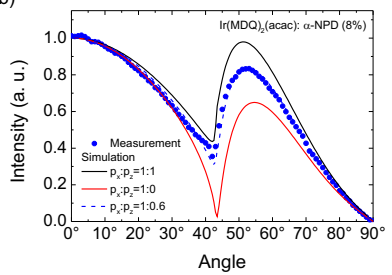

Figure 30 Angular dependent p-polarized photoluminescence intensity of simplified layer stacks on glass substrates with two different phosphorescent dyes: (a) $\operatorname{Ir}(\text { ppy })_{3}$ embedded in a CBP matrix (wavelength $510 \mathrm{~nm}$ ) and (b) $\operatorname{Ir}(\mathrm{MDQ})_{2}$ (acac) in an $\alpha$ NPD matrix (wavelength $610 \mathrm{~nm}$ ). (For details of the experiment see Ref. [105]). The comparison to simulations for exclusively horizontal and fully random emitter orientation reveals isotropic emitter orientation in case (a), while case (b) shows a predominantly horizontal orientation with a ratio of 2:(0.6 \pm 0.1$)$ parallel $\left(p_{x}+p_{y}\right)$ vs. perpendicular $\left(p_{z}\right)$ emitters, in good agreement with Ref. [68].
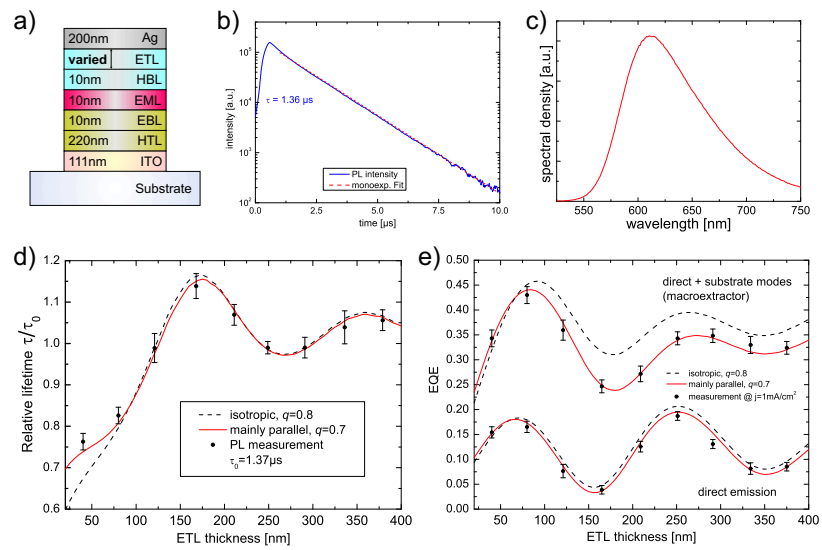

Figure 31 (a) Structure of the red phosphorescent OLED stack containing $\operatorname{Ir}(\mathrm{MDQ})_{2}$ (acac) in an $\alpha$-NPD matrix as EML, (b) an example of a phosphorescence lifetime measurement for an ETL thickness of $249 \mathrm{~nm}$ and (c) the electroluminescence spectrum used for simulation. (d) Measured phosphorescence lifetimes normalized to the intrinsic value $\tau_{0}=1.37 \mu$ s together with simulated curves for different emitter orientation: isotropic with $q=0.8$ and mainly horizontally oriented with an anisotropy parameter $\theta=0.63 / 2.63=0.24$ and $q=0.7$. (e) Measured EQE values without and with a macro-extractor at a current density of $j=1 \mathrm{~mA} / \mathrm{cm}^{2}$ are compared to optical simulations for both situations, clearly confirming the non-isotropic orientation of this emitter system. (For details see Ref. [58]).

As described in detail in Ref. [67], we have compared OLEDs incorporating two blue emitting fluorescent dyes (BDASBi and PEBA, see Fig. 3) exhibiting different emitter orientation with respect to their external quantum efficiency. In the actual OLEDs investigated by us the emitter orientation is not perfectly horizontal and the emitter quantum efficiency is less than unity. Nevertheless, the ex- perimentally observed increase in EQE with respect to the isotropic reference OLED can consistently be explained by the preferentially horizontal emitter orientation in case of the rod-like BDASBi molecule as emitting dye. Meanwhile, other groups have also found evidence for a possible contribution of non-isotropic emitter orientation in fluorescent OLEDs with efficiencies beyond the spin-statistical limit [30,31].

Recently, we have extended these studies towards highly efficient phosphorescent emitters. As expected, symmetrically substituted metal-organic emitter complexes such as $\operatorname{Ir}(\mathrm{ppy})_{3}$ have random emitter orientation when doped in a CBP matrix (see Fig. 30(a)), however, in the case of complexes with different ligands there is evidence for non-isotropic emitter orientation. For example, the well-known red phosphorescent emitter system $\operatorname{Ir}(\mathrm{MDQ})_{2}$ (acac) doped into an $\alpha$-NPD matrix also exhibits predominantly horizontal orientation of the emitting dipoles (see Fig. 30(b) and Ref. [68]). If this circumstance is ignored, efficiency analysis based on the assumption of isotropic emitter orientation will lead to an overestimation of the radiative quantum efficiency $[108,109]$, as has been worked out in detail in Ref. [58]. Fig. 31 shows the OLED stack, on which we have performed a comprehensive efficiency analysis combining phosphorescence lifetime and EQE measurements as function of the ETL thickness, as described before in section 4.5 for an $\operatorname{Ir}(\text { ppy })_{3}$ based green OLED. Both data sets show that a consistent description can only be obtained, if non-isotropic emitter orientation is taken into account. Moreover, optical simulations clearly reveal that horizontally oriented phosphorescent emitters with internal radiative quantum efficiencies close to unity have the potential to achieve EQE values exceeding 30\% for direct emission to air and up to $70 \%$, if all of the substrate emission can be extracted $[58,110]$.

6 Summary and Outlook In conclusion, we have shown that optical modelling is an indispensable tool not only for optimization of OLED light outcoupling, but also for a comprehensive efficiency analysis. In the latter case, simulations have to be combined with systematic parameter variation of the OLED layer stack, e.g. the distance of the emission zone to the highly reflecting cathode. In this way it is possible to separate microcavity effects on the radiative rate, determining the effective radiative quantum efficiency $q_{\mathrm{eff}}$, from a redistribution of energy between different optical channels, affecting the light outcoupling term $\eta_{\text {out }}$. We find a significant reduction of $q_{\text {eff }}$ under typical OLED operating conditions, which has to be considered in the stack design.

Furthermore, we have presented approaches for enhancing $\eta_{\text {out }}$, with special emphasis on the reduction of the surface plasmon loss channel. Beside scattering approaches and high-index coupling, the control of the emitter orientation has been identified as a particularly powerful handle to obtain this goal. Remarkably, even dye-doped 
small-molecule phosphorescent emitter systems prepared by vacuum deposition can exhibit pronounced anisotropic orientation distributions of their optical transition dipole moments.

Thus, after more than 20 years of research on OLEDs the microscopic understanding of the underlying processes has progressed substantially. With respect to the expression for the external quantum efficiency (Eq. 1) put forward by Tsutsui in 1997 [24] we now have a sound understanding of all four factors allowing for quantitative calculations of OLED efficiencies and predictions towards the potential in improvement of it by newly developed OLED materials, structures and concepts. Moreover, these methods are the basis for a reliable experimental determination of the relevant factors for the OLED efficiency. Nevertheless, the development is ongoing and important new physical concepts, such as the importance and the implications of disorder, are still being elucidated and integrated into OLED modelling.

By now, commercially available white OLEDs are specified with luminous efficacies of about $25 \mathrm{~lm} / \mathrm{W}$ [6], i.e. they are comparable to the best halogen lamps. However, several laboratories have reported values exceeding $60 \mathrm{~lm} / \mathrm{W}$ for devices with good colour compliance being currently under development [10]. Of course there is room for improvement regarding the impressive numbers obtained with inorganic LEDs reaching more than 100 $\mathrm{lm} / \mathrm{W}$. But as already mentioned, the comparison to them is of limited relevance. A strong benefit of OLEDs is their unique form factor, with the light being distributed homogeneously over large area and thus being glare-free. Hence, (almost) no additional fixtures are needed: the OLED already is the luminaire!

Beyond general lighting, the compatibility of organic materials with various kinds of substrates and the ease of processing could open possibilities for new appliances in other fields as well. In particular the topics relevant for light extraction could have fruitful overlap with other fields of optics, e.g. cavity physics, photonic crystals, and plasmonics - and we are still waiting to see the electrically pumped organic laser diode.

Acknowledgements The authors thank the German Ministry of Education and Research (BMBF) for funding within the TOPAS project (FKZ 13N10474). J.F. acknowledges financial support by the Elite Network of Bavaria through the graduate school Materials Science of Complex Interfaces (CompInt) and C.M. by the Bayerische Forschungsstiftung.

We are grateful to colleagues from Osram Opto Semiconductors (Regensburg, Germany) and Fraunhofer Institute for Applied Optics and Precision Engineering (Jena, Germany) as well as the groups of P. Lugli (TU Munich, Germany), C. Adachi (Kyushu University, Japan) and D. Yokoyama (now at Yamagata University, Japan) for their contributions to parts of this work.

\section{References}

[1] Colin J. Humphreys. Solid-state lighting. MRS Bulletin, Special Issue "Harnessing Materials for Energy" (April
2008), 33:459-470, 2008.

[2] M. Pope, H. P. Kallmann, and P. Magnante. Electroluminescence in organic crystals. J. Chem. Phys., 38(8):20422043, 1963.

[3] W. Helfrich and W. G. Schneider. Recombination radiation in anthracene crystals. Phys. Rev. Lett., 14(7):229-231, 1965.

[4] C. W. Tang and S. A. VanSlyke. Organic electroluminescent diodes. Appl. Phys. Lett., 51(12):913-915, 1987.

[5] J.H. Burroughes, D.D.C. Bradley, A.R. Brown, R.N. Marks, K. Mackay, R.H. Friend, P.L. Burns, and A.B. Holmes. Light-emitting diodes based on conjugated polymers. Nature, 347:539, 1990.

[6] Osram and Philips. Commercial white OLEDs are offered since 2010, e.g. by Osram and Philips (see www.osram.com and www.lighting.philips.com).

[7] B.W. D'Andrade and S.R. Forrest. White organic lightemitting devices for solid-state lighting. Advanced Materials, 16(18):1585-1595, 2004.

[8] Gregor Schwartz, Sebastian Reineke, Thomas Conrad Rosenow, Karsten Walzer, and Karl Leo. Triplet harvesting in hybrid white organic light-emitting diodes. Advanced Functional Materials, 19(9):1319-1333, 2009.

[9] Malte C. Gather, Anne Köhnen, and Klaus Meerholz. White organic light-emitting diodes. Advanced Materials, 23(2):233-248, 2011.

[10] Yuan-Sheng Tyan. Organic light-emitting-diode lighting overview. J. Photon. Energy, 1:011009, 2011.

[11] Lixin Xiao, Zhijian Chen, Bo Qu, Jiaxiu Luo, Sheng Kong, Qihuang Gong, and Junji Kido. Recent progresses on materials for electrophosphorescent organic light-emitting devices. Advanced Materials, 23(8):926-952, 2011.

[12] L. S. Hung, C. W. Tang, and M. G. Mason. Enhanced electron injection in organic electroluminescence devices using an $\mathrm{Al} / \mathrm{LiF}$ electrode. Appl. Phys. Lett., 70(2):152-154, 1997.

[13] R. Coehoorn and S. L. M. van Mensfoort. Effects of disorder on the current density and recombination profile in organic light-emitting diodes. Phys. Rev. B, 80:085302, 2009.

[14] J. Cottaar, R. Coehoorn, and P.A. Bobbert. Modeling of charge transport across disordered organic heterojunctions. Organic Electronics, 13(4):667 - 672, 2012.

[15] M. Kuik, L. J. A. Koster, G. A. H. Wetzelaer, and P. W. M. Blom. Trap-assisted recombination in disordered organic semiconductors. Phys. Rev. Lett., 107:256805, 2011.

[16] N. Koch. Organic electronic devices and their functional interfaces. ChemPhysChem, 8:1438, 2007.

[17] S. L. M. van Mensfoort, M. Carvelli, M. Megens, D. Wehenkel, M. Bartyzel, H. Greiner, R. A. J. Janssen, and R. Coehoorn. Measuring the light emission profile in organic light-emitting diodes with nanometre spatial resolution. Nature Photonics 4, 329 - 335 (2010), 4:329-335, 2010.

[18] M. Pfeiffer, S.R. Forrest, K. Leo, and M.E. Thompson. Electrophosphorescent pin organic light-emitting devices for very-high-efficiency flat-panel displays. Advanced Materials, 14(22):1633-1636, 2002.

[19] M. A. Baldo, D. F. O'Brien, Y. You, A. Shoustikov, S. Sibley, M. E. Thompson, and S. R. Forrest. Highly efficient 
phosphorescent emission from organic electroluminescent devices. Nature, 395(6698):151-154, 1998.

[20] Sebastian Reineke, Frank Lindner, Gregor Schwartz, Nico Seidler, Karsten Walzer, Björn Lüssem, and Karl Leo. White organic light-emitting diodes with fluorescent tube efficiency. Nature, 459(7244):234-238, 2009.

[21] Z. B. Wang, M. G. Helander, J. Qiu, D. P. Puzzo, M. T. Greiner, Z. M. Hudson, S. Wang, Z. W. Liu, and Z. H. Lu. Unlocking the full potential of organic light-emitting diodes on flexible plastic. Nature Photonics, 5:753-757, 2011.

[22] J. Kido, H. Hayase, K. Hongawa, K. Nagai, and K. Okuyama. Bright red light-emitting organic electroluminescent devices having a europium complex as an emitter. Appl. Phys. Lett., 65:2124, 1994.

[23] Franky So, Benjamin Krummacher, Mathew K. Mathai, Dmitry Poplavskyy, Stelios A. Choulis, and Vi-En Choong. Recent progress in solution processable organic light emitting devices. J. Appl. Phys., 102(9):091101-21, 2007.

[24] T. Tsutsui, E. Aminaka, C. P. Lin, and D. U. Kim. Extended molecular design concept of molecular materials for electroluminescence: sublimed-dye films, molecularly doped polymers and polymers with chromophores. Phil. Trans. R. Soc. Lond. A, 355(1725):801-814, 1997.

[25] Chihaya Adachi, Marc A. Baldo, Mark E. Thompson, and Stephen R. Forrest. Nearly 100\% internal phosphorescence efficiency in an organic light-emitting device. J. Appl. Phys., 90(10):5048-5051, 2001.

[26] M. A. Baldo, D. F. O'Brien, M. E. Thompson, and S. R. Forrest. Excitonic singlet-triplet ratio in a semiconducting organic thin film. Phys. Rev. B, 60(20):14422-14428, 1999.

[27] M. Segal, M. A. Baldo, R. J. Holmes, S. R. Forrest, and Z. G. Soos. Excitonic singlet-triplet ratios in molecular and polymeric organic materials. Phys. Rev. B, 68(7):07521114, 2003.

[28] D. Y. Kondakov, T. D. Pawlik, T. K. Hatwar, and J. P. Spindler. Triplet annihilation exceeding spin statistical limit in highly efficient fluorescent organic light-emitting diodes. J. Appl. Phys., 106(12):124510-7, 2009.

[29] Yichun Luo and Hany Aziz. Correlation between triplettriplet annihilation and electroluminescence efficiency in doped fluorescent organic light-emitting devices. $A d v$. Funct. Mater., 20(8):1285-1293, 2010.

[30] Daisuke Yokoyama, Youngil Park, Beomjin Kim, Sookang Kim, Yong-Jin Pu, Junji Kido, and Jongwook Park. Dual efficiency enhancement by delayed fluorescence and dipole orientation in high-efficiency fluorescent organic lightemitting diodes. Applied Physics Letters, 99(12):123303, 2011.

[31] Yong-Jin Pu, Go Nakata, Fumiya Satoh, Hisahiro Sasabe, Daisuke Yokoyama, , and Junji Kido. Optimizing the charge balance of fluorescent organic light-emitting devices to achieve high external quantum efficiency beyond the conventional upper limit. Advanced Materials, 24:17651770, 2012.

[32] Ayataka Endo, Keigo Sato, Kazuaki Yoshimura, Takahiro Kai, Atsushi Kawada, Hiroshi Miyazaki, and Chihaya Adachi. Efficient up-conversion of triplet excitons into a singlet state and its application for organic light emitting diodes. Applied Physics Letters, 98(8):083302, 2011.
[33] Kenichi Goushi, Kou Yoshida, Keigo Sato, and Chihaya Adachi. Organic light-emitting diodes employing efficient reverse intersystem crossing for triplet-to-singlet state conversion. Nature Photonics, 6:253258, 2012.

[34] M. Wohlgenannt, Kunj Tandon, S. Mazumdar, S. Ramasesha, and Z. V. Vardeny. Formation cross-sections of singlet and triplet excitons in $\pi$-conjugated polymers. Nature, 409(6819):494-497, 2001.

[35] Z. Shuai, D. Beljonne, R. J. Silbey, and J. L. Brédas. Singlet and triplet exciton formation rates in conjugated polymer light-emitting diodes. Phys. Rev. Lett., 84(1):131-134, 2000.

[36] C. Rothe, S. M. King, and A. P. Monkman. Direct measurement of the singlet generation yield in polymer lightemitting diodes. Phys. Rev. Lett., 97(7):076602-4, 2006.

[37] S. L. M. van Mensfoort, J. Billen, M. Carvelli, S. I. E. Vulto, R. A. J. Janssen, and R. Coehoorn. Predictive modeling of the current density and radiative recombination in blue polymer-based light-emitting diodes. J. Appl. Phys., 109:064502, 2011.

[38] J. S. Wilson, A. S. Dhoot, A. J. A. B. Seeley, M. S. Khan, A. Köhler, and R. H. Friend. Spin-dependent exciton formation in $\pi$-conjugated compounds. Nature, 413(6858):828-831, 2001.

[39] Benjamin C. Krummacher, Stefan Nowy, Jörg Frischeisen, Markus Klein, and Wolfgang Brütting. Efficiency analysis of organic light-emitting diodes based on optical simulation. Org. Electron., 10(3):478-485, 2009.

[40] Stefan Nowy, Benjamin C. Krummacher, Jörg Frischeisen, Nils A. Reinke, and Wolfgang Brütting. Light extraction and optical loss mechanisms in organic light-emitting diodes: Influence of the emitter quantum efficiency. J. Appl. Phys., 104(12):123109-9, 2008.

[41] H. Becker, S. E. Burns, and R. H. Friend. Effect of metal films on the photoluminescence and electroluminescence of conjugated polymers. Phys. Rev. B, 56(4):1893-1905, 1997.

[42] J. A. E. Wasey, A. Safonov, I. D. W. Samuel, and W. L. Barnes. Efficiency of radiative emission from thin films of a light-emitting conjugated polymer. Phys. Rev. B, 64(20):205201-9, 2001.

[43] N. C. Giebink and S. R. Forrest. Quantum efficiency rolloff at high brightness in fluorescent and phosphorescent organic light emitting diodes. Physical Review B, 77:235215, 2008.

[44] Neil C. Greenham, Richard H. Friend, and Donal D. C. Bradley. Angular dependence of the emission from a conjugated polymer light-emitting diode: Implications for efficiency calculations. Adv. Mater., 6(6):491-494, 1994.

[45] L. H. Smith, J. A. E. Wasey, I. D. W. Samuel, and W. L. Barnes. Light out-coupling efficiencies of organic lightemitting diode structures and the effect of photoluminescence quantum yield. Adv. Funct. Mater., 15(11):18391844, 2005.

[46] Stefan Nowy, Jörg Frischeisen, and Wolfgang Brütting. Simulation based optimization of light-outcoupling in organic light-emitting diodes. Proc. SPIE, 7415(1):74151C9, 2009. 
[47] Kanchan Saxena, V.K. Jain, and Dalip Singh Mehta. A review on the light extraction techniques in organic electroluminescent devices. Opt. Mater., 32(1):221 - 233, 2009.

[48] E.M. Purcell. Spontaneous emission probabilities at radio frequencies. Phys. Rev., 69:681, 1946.

[49] K. H. Drexhage. Influence of a dielectric interface on fluorescence decay time. J. Lumin., 1-2:693-701, 1970.

[50] K. H. Drexhage. Interaction of light with monomolecular dye layers. Prog. Optics, 12:165, 1974.

[51] R. R. Chance, A. Prock, and R. Silbey. Lifetime of an emitting molecule near a partially reflecting surface. J. Chem. Phys., 60(7):2744-2748, 1974.

[52] R. R. Chance, A. Prock, and R. Silbey. Molecular fluorescence and energy transfer near interfaces. Adv. Chem. Phys., 37:1-65, 1978.

[53] W. Lukosz. Light emission by multipole sources in thin layers. i. radiation patterns of electric and magnetic dipoles. J. Opt. Soc. Am, 71:744-754, 1981.

[54] K. A. Neyts. Simulation of light emission from thin-film microcavities. J. Opt. Soc. Am. A, 15:962-971, 1998.

[55] W. L. Barnes. Fluorescence near interfaces: The role of photonic mode density. J. Mod. Opt., 45(4):661-699, 1998.

[56] J. A. E. Wasey and W. Barnes. Efficiency of spontaneous emission from planar microcavities. J. Mod. Opt., 47:725$741,2000$.

[57] Malte C. Gather, Michael Flämmich, Norbert Danz, Dirk Michaelis, and Klaus Meerholz. Measuring the profile of the emission zone in polymeric organic light-emitting diodes. Appl. Phys. Lett., 94(26):263301, 2009.

[58] T. D. Schmidt, D. S. Setz, M. Flämmich, J. Frischeisen, D. Michaelis, B. Krummacher, N. Danz, and W. Brütting. Evidence for non-isotropic emitter orientation in a red phosphorescent organic light-emitting diode and its implications for determining the emitter's radiative quantum efficiency. Appl. Phys. Lett., 99:163302, 2011.

[59] Michael Flämmich, Malte C. Gather, Norbert Danz, Dirk Michaelis, Andreas H. Braeuer, Klaus Meerholz, and Andreas Tuennermann. Orientation of emissive dipoles in OLEDs: Quantitative in situ analysis. Organic Electronics, 11(6):1039-1046, 2010.

[60] Michael Flämmich, Dirk Michaelis, and Norbert Danz. Accessing oled emitter properties by radiation pattern analyses. Organic Electronics, 12(1):83-91, 2011.

[61] Chun-Liang Lin, Ting-Yi Cho, Chih-Hao Chang, and Chung-Chih Wu. Enhancing light outcoupling of organic light-emitting devices by locating emitters around the second antinode of the reflective metal electrode. Appl. Phys. Lett., 88(8):081114-3, 2006.

[62] K. Celebi, T. D. Heidel, and M. A. Baldo. Simplified calculation of dipole energy transport in a multilayer stack using dyadic Green's functions. Opt. Express, 15(4):1762-1772, 2007.

[63] S.-Y Kim and J.-J. Kim. Outcoupling effciency of organic light emitting diodes and the effect of ito thickness. Organic Electronics, 11:1010-1015, 2010.

[64] M. Flämmich, M. C. Gather, N. Danz, D. Michaelis, and K. Meerholz. In situ measurement of the internal luminescence quantum efficiency in organic light-emitting diodes. Applied Physics Letters, 95:263306, 2009.
[65] Daniel S. Setz, Tobias D. Schmidt, Michael Flämmich, Stefan Nowy, Jörg Frischeisen, Benjamin C. Krummacher, Thomas Dobbertin, Karsten Heuser, Dirk Michaelis, Norbert Danz, Wolfgang Brütting, and Albrecht Winnacker. Comprehensive efficiency analysis of organic lightemitting devices. J. Photon. Energy, 1(1):011006, 2011.

[66] M. A. Baldo, S. Lamansky, P. E. Burrows, M. E. Thompson, and S. R. Forrest. Very high-efficiency green organic light-emitting devices based on electrophosphorescence. Appl. Phys. Lett., 75(1):4-6, 1999.

[67] Jörg Frischeisen, Daisuke Yokoyama, Ayataka Endo, Chihaya Adachi, and Wolfgang Brütting. Increased light outcoupling efficiency in dye-doped small molecule organic light-emitting diodes with horizontally oriented emitters. Organic Electronics, 12(5):809-817, 2011.

[68] Michael Flämmich, Jörg Frischeisen, Daniel S. Setz, Dirk Michaelis, Benjamin C. Krummacher, Tobias D. Schmidt, Wolfgang Brütting, and Norbert Danz. Oriented phosphorescent emitters boost oled efficiency. Org. Electron., 12:1663, 2011

[69] K. Meerholz and D. C. Müller. Outsmarting waveguide losses in thin-film light-emitting diodes. Adv. Funct. Mater, 11(4):251-253, 2001.

[70] H. Greiner. Light extraction from organic light emitting diode substrates: simulation and experiment. Jpn. J. Appl. Phys., 46:4125-4137, 2007.

[71] H. Greiner. Light extraction from OLEDs. OLED100 Summer School (Krutyn, June 2010, unpublished).

[72] Jens Meyer, Thomas Winkler, Sami Hamwi, Stephan Schmale, Hans-Hermann Johannes, Thomas Weimann, Peter Hinze, Wolfgang Kowalsky, and Thomas Riedl. Transparent inverted organic light-emitting diodes with a tungsten oxide buffer layer. Adv. Mater, 20(20):3839-3843, 2008.

[73] Mukul Agrawal, Yiru Sun, Stephen R. Forrest, and Peter Peumans. Enhanced outcoupling from organic lightemitting diodes using aperiodic dielectric mirrors. Appl. Phys. Lett., 90(24):241112, 2007.

[74] H. Riel, S. Karg, T. Beierlein, B. Ruhstaller, and W. Rieß. Phosphorescent top-emitting organic light-emitting devices with improved light outcoupling. Appl. Phys. Lett., 82(3):466-468, 2003.

[75] H. Riel, S. Karg, T. Beierlein, W. Riess, and K. Neyts. Tuning the emission characteristics of top-emitting organic light-emitting devices by means of a dielectric capping layer: An experimental and theoretical study. J. Appl. Phys., 94(8):5290-5296, 2003.

[76] Michael Thomschke, Robert Nitsche, Mauro Furno, and Karl Leo. Optimized efficiency and angular emission characteristics of white top-emitting organic electroluminescent diodes. Appl. Phys. Lett., 94(8):083303, 2009.

[77] Simone Hofmann, Michael Thomschke, Patricia Freitag, Mauro Furno, Björn Lüssem, and Karl Leo. Top-emitting organic light-emitting diodes: Influence of cavity design. Appl. Phys. Lett., 97(25):253308, 2010.

[78] L.H. Smith and W.L. Barnes. Using a low-index host layer to increase emission from organic light-emitting diode structures. Org. Electron., 7(6):490-494, 2006.

[79] T. Nakamura, N. Tsutsumi, N. Juni, and H. Fujii. Thin-film waveguiding mode light extraction in organic electrolumi- 
nescent device using high refractive index substrate. Journal of Applied Physics, 97:054505, 2005.

[80] Georg Gaertner and Horst Greiner. Light extraction from OLEDs with (high) index matched glass substrates. Proc. SPIE, 6999(1):69992T-11, 2008.

[81] Saso Mladenovski, Kristiaan Neyts, Domagoj Pavicic, Ansgar Werner, and Carsten Rothe. Exceptionally efficient organic light emitting devices using high refractive index substrates. Opt. Express, 17(9):7562-7570, 2009.

[82] C. F. Madigan, M.-H. Lu, and J. C. Sturm. Improvement of output coupling efficiency of organic light-emitting diodes by backside substrate modification. Appl. Phys. Lett., 76(13):1650-1652, 2000.

[83] S. Möller and S. R. Forrest. Improved light out-coupling in organic light emitting diodes employing ordered microlens arrays. J. Appl. Phys., 91(5):3324-3327, 2002.

[84] Takashi Yamasaki, Kazuhiro Sumioka, and Tetsuo Tsutsui. Organic light-emitting device with an ordered monolayer of silica microspheres as a scattering medium. Appl. Phys. Lett., 76(10):1243-1245, 2000.

[85] Boris Riedel, Inga Kaiser, Julian Hauss, Uli Lemmer, and Martina Gerken. Improving the outcoupling efficiency of indium-tin-oxide-free organic light-emitting diodes via rough internal interfaces. Optics Express, 18(23):A631A639, 2010.

[86] John M. Lupton, Benjamin J. Matterson, Ifor D. W. Samuel, Michael J. Jory, and William L. Barnes. Bragg scattering from periodically microstructured light emitting diodes. Appl. Phys. Lett., 77(21):3340-3342, 2000.

[87] Yiru Sun and Stephen R. Forrest. Enhanced light outcoupling of organic light-emitting devices using embedded low-index grids. Nature Photon., 2(8):483-487, 2008.

[88] Won Hoe Koo, Soon Moon Jeong, Fumito Araoka, Ken Ishikawa, Suzushi Nishimura, Takehiro Toyooka, and Hideo Takezoe. Light extraction from organic lightemitting diodes enhanced by spontaneously formed buckles. Nature Photon., 4(4):222-226, 2010.

[89] S. Wedge, I. R. Hooper, I. Sage, and W. L. Barnes. Light emission through a corrugated metal film: The role of cross-coupled surface plasmon polaritons. Phys. Rev. B, 69(24):245418-8, 2004.

[90] Heinz Raether. Surface Plasmons on Smooth and Rough Surfaces and on Gratings. Springer, 1988.

[91] Stefan Alexander Maier. Plasmonics: Fundamentals and Applications. Springer, 2007.

[92] Harry A. Atwater. The promise of plasmonics. Scientific American, 296(4):56-63, 2007.

[93] P. Andrew and W. L. Barnes. Energy transfer across a metal film mediated by surface plasmon polaritons. Science, 306(5698):1002-1005, 2004.

[94] Jörg Frischeisen, Bert J. Scholz, Benedikt J. Arndt, Tobias D. Schmidt, Robert Gehlhaar, Chihaya Adachi, and Wolfgang Brütting. Strategies for light extraction from surface plasmons in organic light-emitting diodes. J. Photon. Energy, 1(1):011004, 2011.

[95] Jörg Frischeisen, Quan Niu, Alaa Abdellah, Jörg B. Kinzel, Robert Gehlhaar, Giuseppe Scarpa, Chihaya Adachi, Paolo Lugli, and Wolfgang Brütting. Light extraction from surface plasmons and waveguide modes in an organic lightemitting layer by nanoimprinted gratings. Opt. Express, 19(S1):A7-A19, 2011.
[96] W. L. Barnes. Electromagnetic crystals for surface plasmon polaritons and the extraction of light from emissive devices. J. Lightwave Technology, 17:2170-2182, 1999.

[97] S. Wedge, A. Giannattasio, and W.L. Barnes. Surface plasmon-polariton mediated emission of light from topemitting organic light-emitting diode type structures. Org. Electron., 8(2-3):136-147, 2007.

[98] Julian Hauss, Boris Riedel, Sebastian Gleiss, Ulf Geyer, Uli Lemmer, and Martina Gerken. Periodic nanostructuring for guided mode extraction in organic light-emitting diodes. $J$. Photon. Energy, 1(1):011012, 2011.

[99] B. J. Scholz, J. Frischeisen, A. Jaeger, D. S. Setz, T. Reusch, and W. Brütting. Extraction of surface plasmons in organic light-emitting diodes via high-index coupling. Opt. Express, 20:A205, 2012.

[100] Erwin Kretschmann. Die Bestimmung optischer Konstanten von Metallen durch Anregung von Oberflächenplasmaschwingungen. Z. Physik, 241:313324, 1971.

[101] G. Winter and W. L. Barnes. Emission of light through thin silver films via near-field coupling to surface plasmon polaritons. Appl. Phys. Lett., 88(5):051109-3, 2006.

[102] W. Brütting, J. Frischeisen, B. J. Scholz, and T. D. Schmidt. More light from organic light-emitting diodes. Europhysics News, 42 (4):20, 2011

[103] Kun-Cheng Tien, Ming-Shiang Lin, You-Heng Lin, ChihHung Tsai, Ming-Hung Shiu, Ming-Chi Wei, Hsing-Chieh Cheng, Chun-Liang Lin, Hao-Wu Lin, and Chung-Chih Wu. Utilizing surface plasmon polariton mediated energy transfer for tunable double-emitting organic light-emitting devices. Org. Electron., 11(3):397 - 406, 2010.

[104] Ji-Seon Kim, Peter K. H. Ho, Neil C. Greenham, and Richard H. Friend. Electroluminescence emission pattern of organic light-emitting diodes: Implications for device efficiency calculations. J. Appl. Phys., 88(2):1073-1081, 2000.

[105] Jörg Frischeisen, Daisuke Yokoyama, Chihaya Adachi, and Wolfgang Brütting. Determination of molecular dipole orientation in doped fluorescent organic thin films by photoluminescence measurements. Appl. Phys. Lett., 96(7):073302, 2010.

[106] W. H. Weber and C. F. Eagen. Energy transfer from an excited dye molecule to the surface plasmons of an adjacent metal. Optics Letters, 4:236-238, 1979.

[107] Daisuke Yokoyama, Akio Sakaguchi, Michio Suzuki, and Chihaya Adachi. Horizontal orientation of linear-shaped organic molecules having bulky substituents in neat and doped vacuum-deposited amorphous films. Org. Electron., 10(1): 127 - 137, 2009.

[108] Rico Meerheim, Mauro Furno, Simone Hofmann, Björn Lüssem, and Karl Leo. Quantification of energy loss mechanisms in organic light-emitting diodes. Appl. Phys. Lett., 97(25):253305, 2010.

[109] Mauro Furno, Rico Meerheim, Simone Hofmann, Björn Lüssem, and Karl Leo. Efficiency and rate of spontaneous emission in organic electroluminescent devices. Phys. Rev. $B, 85: 115205,2012$.

[110] Tobias D. Schmidt, Michael Flämmich, Bert J. Scholz, Dirk Michaelis, Christian Mayr, Norbert Danz, and Wolfgang 
Brütting. Non-isotropic emitter orientation and its implications for efficiency analysis of organic light-emitting diodes. Proc. SPIE, 8435:843513, 2012. 